Geometry $\&$ Topology

Volume 8 (2004) 1-34

Published: 18 January 2004

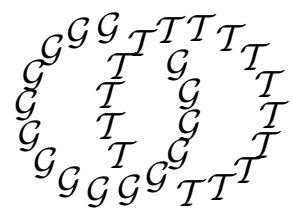

\title{
Modular circle quotients and PL limit sets
}

\author{
RICHARD Evan SchWARTZ \\ Department of Mathematics, University of Maryland \\ College Park, MD 20742, USA \\ Email: res@math.umd.edu
}

\begin{abstract}
We say that a collection $\Gamma$ of geodesics in the hyperbolic plane $\boldsymbol{H}^{2}$ is a modular pattern if $\Gamma$ is invariant under the modular group $P S L_{2}(\boldsymbol{Z})$, if there are only finitely many $P S L_{2}(\boldsymbol{Z})$-equivalence classes of geodesics in $\Gamma$, and if each geodesic in $\Gamma$ is stabilized by an infinite order subgroup of $P S L_{2}(\boldsymbol{Z})$. For instance, any finite union of closed geodesics on the modular orbifold $\boldsymbol{H}^{2} / P S L_{2}(\boldsymbol{Z})$ lifts to a modular pattern. Let $S^{1}$ be the ideal boundary of $\boldsymbol{H}^{2}$. Given two points $p, q \in S^{1}$ we write $p \sim q$ if $p$ and $q$ are the endpoints of a geodesic in $\Gamma$. (In particular $p \sim p$.) We will see in section 3.2 that $\sim$ is an equivalence relation. We let $Q_{\Gamma}=S^{1} / \sim$ be the quotient space. We call $Q_{\Gamma}$ a modular circle quotient. In this paper we will give a sense of what modular circle quotients "look like" by realizing them as limit sets of piecewise-linear group actions
\end{abstract}

\section{AMS Classification numbers Primary: $57 \mathrm{~S} 30$}

Secondary: 54E99, 51M15

Keywords: Modular group, geodesic patterns, limit sets, representations

Proposed: David Gabai

Seconded: Martin Bridson, Walter Neumann
Received: 4 February 2003

Accepted: 13 January 2004 


\section{Introduction}

In this paper we address the question: What does a tennis racket look like if it is strung so tightly that the individual strings collapse into points? Rather than consider the expensive disasters produced by an actual experiment we will consider related theoretical objects called modular circle quotients.

We say that a collection $\Gamma$ of geodesics in the hyperbolic plane $\boldsymbol{H}^{2}$ is a modular pattern if $\Gamma$ is invariant under the modular group $P S L_{2}(\boldsymbol{Z})$, if there are only finitely many $P S L_{2}(\boldsymbol{Z})$-equivalence classes of geodesics in $\Gamma$, and if each geodesic in $\Gamma$ is stabilized by an infinite order subgroup of $P S L_{2}(\boldsymbol{Z})$. For instance, any finite union of closed geodesics on the modular orbifold $\boldsymbol{H}^{2} / P S L_{2}(\boldsymbol{Z})$ lifts to a modular pattern. Let $S^{1}$ be the ideal boundary of $\boldsymbol{H}^{2}$. Given two points $p, q \in S^{1}$ we write $p \sim q$ if $p$ and $q$ are the endpoints of a geodesic in $\Gamma$. (In particular $p \sim p$.) We will see in section 3.2 that $\sim$ is an equivalence relation. We let $Q_{\Gamma}=S^{1} / \sim$ be the quotient space. We call $Q_{\Gamma}$ a modular circle quotient.

In [7] we encountered a certain modular circle quotient as the limit set of a special representation of $P S L_{2}(\boldsymbol{Z})$ into $P U(2,1)$, the group of complex projective automorphisms of the 3 -sphere $S^{3}$. In 8 we embedded some related circle quotients into $S^{3}$. In this paper we will treat all the modular circle quotients, motivated by the constructions in [8] but starting from scratch. Our aim is to give a sense of what they look like, by realizing them as limit sets of piecewise linear group actions.

\subsection{Statement of results}

Let $\Gamma$ be a modular pattern of geodesics. As we explain in section 3.1, there is a well-known tiling of $\boldsymbol{H}^{2}$ by ideal triangles which is invariant under the action of $P S L_{2}(\boldsymbol{Z})$. We call this tiling the modular tiling. We define $|\Gamma|$ to be one more than the number of geodesics in $\Gamma$ which intersect a given edge of the modular tiling. We will see in section 3.2 that this number is finite. $|\Gamma|$ is independent of the choice of edge, by symmetry.

Let $S^{n}$ be the $n$-sphere. Our model for $S^{n}$ is the double of an $n$-simplex: $S^{n}=\Delta_{+} \cup \Delta_{-}$, where $\Delta_{+}$and $\Delta_{-}$are two copies of an $n$-simplex, glued along their boundaries. A simplex of $S^{n}$ is a sub-simplex of either $\Delta_{+}$or $\Delta_{-}$. Say that a punctured simplex of $S^{n}$ is a simplex with its vertices deleted.

A homeomorphism $h$ of $S^{n}$ is piecewise linear (or PL) if there is some triangulation of $S^{n}$ into finitely many simplices such that $h$ is affine when restricted 
to each simplex in the triangulation. The set of PL homeomorphisms of $S^{n}$ forms a topological group $\mathrm{PL}\left(S^{n}\right)$ equipped with the compact-open topology. Let $H \subset \mathrm{PL}\left(S^{n}\right)$ be a subgroup. A compact subset $\Lambda \subset S^{n}$ is the limit set of $H$ if $H$ acts properly discontinuously on $S^{n}-\Lambda$ and minimally on $\Lambda$. Thus $H(x)$ is dense in $\Lambda$ for every $x \in \Lambda$ and for any compact $K \subset S^{n}-\Lambda$, the set $\{h \in H \mid h(K) \cap K \neq \emptyset\}$ is finite.

Theorem 1.1 Let $n=|\Gamma|$. There is an embedding $i: Q_{\Gamma} \rightarrow S^{n}$ and a monomorphism $\rho: P S L_{2}(\boldsymbol{Z}) \rightarrow \mathrm{PL}\left(S^{n}\right)$ such that $i\left(Q_{\Gamma}\right)$ is the limit set of $\rho\left(P S L_{2}(\boldsymbol{Z})\right)$. There is a $\rho\left(P S L_{2}(\boldsymbol{Z})\right)$-invariant partition of $S^{n}-i\left(Q_{\Gamma}\right)$ into punctured simplices, the vertices of which are densely contained in $i\left(Q_{\Gamma}\right)$.

One generalization of a modular pattern is a $P S L_{2}(\boldsymbol{Z})$-invariant map $f: \Gamma \rightarrow$ $(0,1]$, where $\Gamma$ is a modular pattern. Let $\square(\Gamma)$ be the space of these maps. Let $C S\left(S^{n}\right)$ be the space of closed subsets of $S^{n}$, given the Hausdorff topology. (Two subsets are close if each is contained in a small tubular neighborhood of the other.) Let $\operatorname{Mon}\left(P S L_{2}(\boldsymbol{Z}), \operatorname{PL}\left(S^{n}\right)\right)$ denote the space of monomorphisms from $P S L_{2}(\boldsymbol{Z})$ into $\mathrm{PL}\left(S^{n}\right)$ given the algebraic topology. (Two monomorphisms are close if they map the generators to nearby elements of $\operatorname{PL}\left(S^{n}\right)$.) The following result organizes all the modular circle quotients based on subpatterns $\Gamma^{\prime}$ of $\Gamma$.

Theorem 1.2 Let $n=|\Gamma|$. There are continuous maps $\Lambda: \square(\Gamma) \rightarrow C S\left(S^{n}\right)$ and $\rho: \square(\Gamma) \rightarrow \operatorname{Mon}\left(P S L_{2}(\boldsymbol{Z}), \operatorname{PL}\left(S^{n}\right)\right)$ such that the following is true for all $f \in \square(\Gamma)$. The set $\Lambda_{f}$ is the limit set of $\rho_{f}\left(P S L_{2}(\boldsymbol{Z})\right)$ and $\Lambda_{f}$ is homeomorphic to $Q_{\Gamma^{\prime}}$, where $\Gamma^{\prime}=f^{-1}(1)$.

The method we use to prove Theorem 1.2 is flexible and allows us to make a statement about more general kinds of circle quotients:

Theorem 1.3 Let $\Gamma^{\prime}$ be the lift to $H^{2}$ of an arbitrary finite union of closed geodesics on a cusped hyperbolic surface $\Sigma$. Let $Q_{\Gamma^{\prime}}$ be the circle quotient based on $\Gamma^{\prime}$. For some $n$ there is an embedding $i: Q_{\Gamma^{\prime}} \rightarrow S^{n}$ and a monomorphism $\rho: \pi_{1}(\Sigma) \rightarrow \mathrm{PL}\left(S^{n}\right)$ such that $i\left(Q_{\Gamma^{\prime}}\right)$ is the limit set of $\rho\left(\pi_{1}(\Sigma)\right)$.

If $\Gamma_{1}$ and $\Gamma_{2}$ are both modular patterns and $\Gamma_{1} \subset \Gamma_{2}$ then we have an inclusion $\square\left(\Gamma_{1}\right) \hookrightarrow \partial \square\left(\Gamma_{2}\right)$. Assuming this inclusion implicitly, we say that a sequence $\left\{f_{m}\right\} \in \square\left(\Gamma_{2}\right)$ degenerates to $f \in \square\left(\Gamma_{1}\right)$ if $f_{m}(\gamma) \rightarrow 0$ for all $\gamma \in \Gamma_{2}-\Gamma_{1}$ and $f_{m}(\gamma) \rightarrow f(\gamma)$ if $\gamma \in \Gamma_{1}$. Let $n_{j}=\left|\Gamma_{j}\right|$ for $j=1,2$. The $n_{2}$-simplex has faces which are $n_{1}$-simplices. The doubles of these $n_{1}$-simplices are copies of $S^{n_{1}}$ contained in $S^{n_{2}}$. We call these copies the natural embeddings of $S^{n_{1}}$ into $S^{n_{2}}$. 
Theorem 1.4 There is a natural embedding $i: S^{n_{1}} \hookrightarrow S^{n_{2}}$ with the following property. Let $\left\{f_{m}\right\} \in \square\left(\Gamma_{2}\right)$ be a sequence which degenerates to $f \in \square\left(\Gamma_{1}\right)$. Then the limit sets $\Lambda_{f_{m}}$ converge to $i\left(\Lambda_{f}\right)$. The restriction of $\rho_{f_{m}}$ to $\Lambda_{f_{m}}$ converges to the action of $i \circ \rho_{f} \circ i^{-1}$ on $i\left(\Lambda_{f}\right)$.

Theorem 1.4 covers one case not explicitly mentioned. In section [5.5 we define a certain standard representation $\rho_{0}: P S L_{2}(\boldsymbol{Z}) \rightarrow P L\left(S^{1}\right)$. If $f_{m}(\gamma) \rightarrow 0$ for all $\gamma \in \Gamma_{2}$ then $\Lambda_{f_{m}}$ converges to a naturally embedded circle $i\left(S^{1}\right)$ and the restriction of $\rho_{f_{m}}$ to $\Lambda_{f_{m}}$ converges to $i \circ \rho_{0} \circ i^{-1}$.

Theorem 1.4 lets us organize all the modular circle quotients into a coherent whole. We define $\square=\bigcup_{\Gamma} \square(\Gamma)$. Let $S^{\infty}$ be the direct limit of $S^{n}$, under the system of natural embeddings. Let $C S\left(S^{\infty}\right)$ denote the set of finite dimensional closed subsets of $S^{\infty}$ equipped with the Hausdorff topology. Then Theorem 1.4 gives a map $\Lambda: \square \rightarrow C S\left(S^{\infty}\right)$ such that $\Lambda_{f}$ is homeomorphic to $Q_{\Gamma^{\prime}}$ and contained in a naturally embedded $|\Gamma|$-dimensional sphere. Here $\Gamma=f^{-1}((0,1])$ and $\Gamma^{\prime}=f^{-1}(1)$. The map $\Lambda$ is continuous when restricted to each finite dimensional subspace of $\square$.

The following construction illustrates the nature of our results. List all the vertices $v_{0}, v_{1}, v_{2} \ldots$ of $\square$ with $v_{0}$ being the vertex corresponding to the 0 map -i.e. the empty pattern. Let $\left\{f_{t} \mid t \in[0, \infty)\right\} \subset \square$ be a continuous path such that $\left.f\right|_{[0, n]}$ is contained in the convex hull of the vertices $v_{0}, \ldots, v_{n}$ and $f_{n}=v_{n}$. Here $n=0,1,2 \ldots$ Then $\Lambda_{0}$ is just the double of a line segment. As $t$ increases $\Lambda_{t}$ continuously and endlessly crinkles up, assuming the topology of every modular circle quotient as it goes.

\subsection{Comparisons and speculation}

Here are some possible connections to our results:

(1) Our constructions here are similar in spirit to our constructions in 9, where we related the modular group to Pappus's theorem and thereby produced discrete representations of the modular group into the group of automorphisms of the real projective plane.

(2) Our Theorem 1.1 seems at least vaguely related to the general results in [2] about embedding the boundaries of hyperbolic groups into $S^{n}$.

(3) Some of the combinatorial ideas underlying our constructions are related to the theory [6] of coding geodesics on the modular surface using their cutting sequences. We can work this out explicitly but don't do it in this paper. 
(4) $\square(\Gamma)$ (with its associated maps) is like a PL version of Teichmuller space. The groups attached to the set $\left\{f \mid f^{-1}(1)=\emptyset\right\}$ are like PL quasi-Fuchsian groups [1, 4] in that their limit sets are topological circles. The other groups are like cusp groups on the boundary of quasi-Fuchsian space.

We elaborate on the fourth item. $\square(\Gamma)$ is both richer and poorer than Teichmuller space. It is richer because it allows for deformations which cannot exist in hyperbolic geometry. There are no nontrivial deformations of the modular group into $\operatorname{Isom}\left(\boldsymbol{H}^{3}\right)$ whereas $\operatorname{dim} \square(\Gamma)$ grows unboundedly with the complexity of $\Gamma$. Indeed, one possible use of our results is that they provide a topological model for degenerating families of representations of punctured surface groups - i.e. finite index subgroups of the modular group - into a Lie group. Such families generally are extremely difficult to construct, let alone study geometrically. Our results give a glimpse of how punctured surface groups might degenerate when non-simple closed geodesics on the surface are pinched.

$\square(\Gamma)$ is poorer than Teichmuller space because it only allows for degenerations which occur by pinching closed geodesics. We don't get things like geometrically infinite limits. It almost goes without saying that $\square(\Gamma)$ is geometrically much poorer than Teichmuller space. It does not enjoy any of the beautiful rigid structure [3] of Teichmuller space.

We wonder how our results transfer to the more rigid setting of a Lie group $G$ acting on a homogeneous space $X$. We think that it ought to be possible sometimes to geometrize our constructions and produce representations of $P S L_{2}(\boldsymbol{Z})$ into $G$ which "realize" our PL representations. The result in [7] is an example of this. On the other hand, we think that there should be strong restrictions on the types of circle quotients for each pair $(G, X)$. A general restriction result would provide a new tool in the study of representations of surface groups into Lie groups, because it would help control the possible degenerations.

As far as we know, all the modular circle quotients are non-planar. At any rate, many of them are non-planar and hence cannot be embedded into $S^{2}$. Probably all of the modular circle quotients can be embedded into $S^{3}$. However, such embeddings would probably be very "distorted" in general. We would like to quantify this distortion, and relate it to the complexity of the modular pattern.

We also wonder about how our results work out for circle quotients based on uniform lattices, but don't have any idea how to proceed. 


\subsection{Some ideas in the proof}

Our main idea is to construct an object we call a modular block (or block for short.) A block is a certain subset $\Omega \subset S^{n}$ equipped with an order $3 \mathrm{PL}$ automorphism $\sigma$. A block is based on a neat partition of the $n$-simplex into $3^{k}-1$ smaller $n$-simplices. Here $k=(n+1) / 2$, with $n$ always being odd. The partition is combinatorially isomorphic to the $k$-fold join of a triangle (which is an $n$-sphere) minus one $n$-simplex. $\Omega$ is obtained by deleting 2 simplices from the partition, so that $\partial \Omega$ consists of 3 non-disjoint $n$-simplex boundaries, called terminals. The remaining $3^{k}-3$ simplices partition $\Omega$ and are permuted by $\sigma$.

We will construct an infinite network of blocks glued together along terminals. The network is essentially tree-like but its fine structure is related to the symbolic coding of geodesics in $\Gamma$. It turns out that $Q_{\Gamma}$ is homeomorphic to the closure of the block vertices. $P S L_{2}(\boldsymbol{Z})$ is represented as a subgroup of the automorphism group of the network. Underlying our block network is a kind of correspondence between some hyperbolic geometry objects related to the modular tiling and some simplicial objects. We call this a simplicial correspondence. The following table summarizes the correspondence.

\begin{tabular}{|c|c|}
\hline hyperbolic object & simplicial object \\
\hline \hline the modular tiling $T$ & modular block network \\
\hline ideal triangle of $T$ & modular block \\
\hline geodesic edge of $T$ & terminal \\
\hline ideal vertex of $T$; geodesic of $\Gamma$ & vertex of a block. \\
\hline circle quotient & closure of the block vertices \\
\hline
\end{tabular}

Here is a more global point of view. We can define an abstract simplicial complex $C(\Gamma)$ whose vertices are elements of $\Gamma \cup V T$. Here $V T$ is the set of ideal vertices of the modular tiling. We say that a subset $S \subset \Gamma \cup V T$ is an abstract simplex if it satisfies the following properties:

(1) There is some ideal triangle $\tau$ of $T$ (not necessarily unique) such that every $s \in S$ is either an ideal vertex of $\tau$ or a geodesic of $\Gamma$ which intersects $\tau$. We say that $\tau$ and $S$ are associated.

(2) If $\tau$ is associated to $S$ and $H_{\tau} \subset P S L_{2}(\boldsymbol{Z})$ is the order-3 stabilizer subgroup of $\tau$ then $S$ does not contain an orbit of $H_{\tau}$. Moreover, $S$ is not stabilized by an order 2 element of $P S L_{2}(\boldsymbol{Z})$. 
Evidently $P S L_{2}(\boldsymbol{Z})$ acts on $C(\Gamma)$. It turns out that the maximal abstract simplices of $C(\Gamma)$ are $n$-dimensional and that $C(\Gamma)$ minus the vertices is a combinatorial $n$-manifold. There are $3^{k}-3$ maximal abstract simplices of $C(\Gamma)$ associated to each $\tau$. Our construction gives an embedding of $C(\Gamma)$ into $S^{n}$ in such a way that these $3^{k}-3$ abstract simplices map to the simplices partitioning the block corresponding to $\tau$. The embedding conjugates the natural action of $P S L_{2}(\boldsymbol{Z})$ on $C(\Gamma)$ to a subgroup of the automorphism group of the block network. The embedding maps the vertex set of $C(G)$ to a dense subset of the limit set.

So far we have sketched the proof of Theorem 1.1. For the remaining results, our idea is to modify the block network by a certain 2-step process. First, we push the blocks apart from each other by attaching collar-like sets, which we call separators, onto the block terminals. Compare Figure 5.1. This process allows the topology of the limit set to vary with the stratum of $\square(\Gamma)$, as in Theorem 1.2. (Theorem 1.3 comes as another application.) Second, we warp the shapes of the individual blocks, to allow the representations associated to $\square\left(\Gamma_{2}\right)$ to degenerate to the representations associated to $\square\left(\Gamma_{1}\right)$, as in Theorem 1.4. The element of $\square(\Gamma)$ determines both the shapes of the warped blocks and the shapes of the separators.

\subsection{Overview of the paper}

We have tried to make this paper completely self-contained. It only relies on a few basic ideas from linear algebra, hyperbolic geometry, and real analysis. We remark to the interested reader that section 2 and 3 makes for a complete, shorter paper in itself, which proves Theorem 1.1. Here is a plan of the rest of paper:

Section 2: Modular blocks, containing: 2.1: The Block Lemma; 2.2: The details; 2.3: 3-dimensional example.

Section 3: Theorem 1.1, containing: 3.1: The modular tiling; 3.2: Modular pattern basics; 3.3: Simplicial correspondences; 3.4: Embedding the quotient; 3.5: Block networks; 3.6: Putting it together.

Section 4: Modified blocks, containing: 4.1: Partial prisms; 4.2: Separators; 4.3: Warped blocks; 4.4: Main construction; 4.5: Degeneration.

Section 5: The rest of the results, containing: 5.1: Modified correspondences; 5.2: Modified block networks; 5.3: Proof of Theorem 1.2 5.4: Proof of Theorem 1.3. 5.5: Proof of Theorem 1.4

\section{References}


Acknowledgements I would like to thank the IHES for their hospitality during the writing of an early version of this paper.

The author is supported by NSF Research Grant DMS-0072607.

\section{Modular blocks}

\subsection{The Block Lemma}

Let $k \geq 2$ be an integer and let $n=2 k-1$. Let $\Delta_{0}$ be an $n$-simplex. We say that a modular block is a set

$$
\Omega=\operatorname{closure}\left(\Delta_{0}-\Delta_{1}-\Delta_{2}\right)
$$

Where $\Delta_{1}, \Delta_{2} \subset \Delta_{0}$ are $n$-simplices with disjoint interiors and

(1) For any indices $i \neq j$ there are $k$ vertices common to $\Delta_{i}$ and $\Delta_{j}$, and $\partial \Delta_{i} \cap \partial \Delta_{j}$ is the convex hull of these common vertices.

(2) There is an order $3 \mathrm{PL}$ automorphism $\sigma: \Omega \rightarrow \Omega$ such that $\sigma$ is affine on $\partial \Delta_{j}$, with orbit $\partial \Delta_{0} \rightarrow \partial \Delta_{1} \rightarrow \partial \Delta_{2} \rightarrow \partial \Delta_{0}$.

We call $\partial \Delta_{j}$ a terminal of $\Omega$ for $j=0,1,2$. We call $\partial \Delta_{0}$ the outer terminal and $\partial \Delta_{1}$ and $\partial \Delta_{2}$ the inner terminals.

Recall from section 1.1 that $S^{n}=\Delta_{+} \cup \Delta_{-}$, where $\Delta_{+}$and $\Delta_{-}$are two copies of a standard $n$-simplex. Our model for $\Delta_{ \pm}$is the convex hull of the standard basis vectors in $\boldsymbol{R}^{n+1}=\boldsymbol{R}^{2 k}$. The goal of this chapter is to prove

Lemma 2.1 (Block Lemma) There exists a modular block whose outer terminal is $\partial \Delta_{+}$.

Proof - modulo some details Let $e_{1}, \ldots, e_{k}$ be the standard basis vectors in $\boldsymbol{R}^{k}$. For any $r \in \boldsymbol{R}$, let $r_{(k)}=(r, \ldots, r) \in \boldsymbol{R}^{k}$. For $j=1, \ldots, k$ we define the following points of $\Delta_{0}=\Delta_{+}$:

$$
A_{j}=\left(e_{j}, 0_{(k)}\right) ; \quad B_{j}=\frac{1}{2 n}\left(2_{(k)}-e_{j}, 2_{(k)}-e_{j}\right) ; \quad C_{j}=\left(0_{k}, e_{j}\right) ;
$$

Let $Y=\left\{Y_{j}\right\}_{j=1}^{k}$ for each letter $Y \in\{A, B, C\}$. Let $\langle\cdot\rangle$ denote the convex hull operation. Note that $\Delta_{0}=\langle A \cup C\rangle$. We define

$$
\Delta_{1}=\langle A \cup B\rangle ; \quad \Delta_{2}=\langle B \cup C\rangle .
$$


The sets $A \cup B$ and $B \cup C$ are bases for $\boldsymbol{R}^{2 k}$. (See Lemma 2.2.) Hence $\Delta_{1}$ and $\Delta_{2}$ are $n$-simplices. Define $u=\left(1_{(k)},-1_{(k)}\right)$. We have $B_{j} \cdot u=0$ for all $j$. Therefore $B$ is contained in the hyperplane $u^{\perp}$. We also have $A_{j} \cdot u=1$ and $C_{j} \cdot u=-1$ for all $j$. Therefore $u^{\perp}$ separates $A$ from $C$. Hence $\Delta_{1} \cap \Delta_{2}=\langle B\rangle$. Since $B \in \operatorname{int}\left(\Delta_{0}\right)$ we have $\partial \Delta_{0} \cap \partial \Delta_{1}=\langle A\rangle$. Likewise $\partial \Delta_{0} \cap \partial \Delta_{2}=\langle C\rangle$. Thus $\Delta_{0}, \Delta_{1}$, and $\Delta_{2}$ satisfy Condition 1 .

Let $X=A \cup B \cup C$. We define $\sigma: X \rightarrow X$ by the action

$$
A_{j} \rightarrow B_{j} \rightarrow C_{j} \rightarrow A_{j} ; \quad j=1, \ldots, k .
$$

Equation 3 implies that $\sigma$ extends to a self-homeomorphism of $\partial \Omega$, which is affine on each terminal. Here $\Omega$ is as in Equation 1 .

Say that a $2 k$-element $S \subset X$ is good if it does not contain any orbits of $\sigma$ and does not equal $A \cup C$. There are $3^{k}-1$ good subsets, two of which are $A \cup B$ and $B \cup C$. Lemma 2.2 below shows that every good set is a basis. We define a good simplex to be a simplex of the form $\langle S\rangle$, where $S$ is a good subset. We can extend the action of $\sigma$ to any individual good simplex other than $\Delta_{1}$ and $\Delta_{2}$ by the rule $\sigma(\langle S\rangle)=\langle\sigma(S)\rangle$.

We will show below that $\Delta_{0}$ is triangulated by the good simplices. That is, $\Delta_{0}=\bigcup_{S}\langle S\rangle$, and for all good simplices $\left\langle S_{1}\right\rangle$ and $\left\langle S_{2}\right\rangle$, we have

$$
\left\langle S_{1}\right\rangle \cap\left\langle S_{2}\right\rangle=\left\langle S_{1} \cap S_{2}\right\rangle .
$$

$\Omega$ is triangulated by the good simplices which are not $\Delta_{1}$ or $\Delta_{2}$, and these are permuted by $\sigma$. Equation 5 implies that all the individual actions of $\sigma$ on good simplices fit together continuously. Hence $\sigma: \Omega \rightarrow \Omega$ satisfies Condition 2 .

\subsection{The details}

For $b \geq 1$ we introduce the $b \times b$ matrix $\Upsilon_{b}$ whose $(i j)$ th entry is 1 if $i=j$ and otherwise 2 . This circulent matrix has the eigenvalue $2 b-1$ with multiplicity 1 and the eigenvalue -1 with multiplicity $b-1$. Therefore

$$
(-1)^{b-1} \operatorname{det}\left(\Upsilon_{b}\right)>0 .
$$

Before proving Lemma 2.2 let's consider a representative example which shows how $\Upsilon_{b}$ arises in our calculations. We take $(k, n)=(3,5)$ and show that the set $S=\left\{A_{1}, B_{1}, B_{2}, C_{2}, A_{3}, C_{3}\right\}$ is a basis for $\boldsymbol{R}^{6}$. Let $M$ be the matrix whose rows are elements of $S$. If some row has a single 1 in the $j$ th spot, and 0 s in all other spots, we change $j$ th spots of all the other rows to 0 . We call this simple row reduction. We use a combination of permutations and simple row 
reductions to show that $\operatorname{det}(M) \neq 0$. Ignoring the factor of $\frac{1}{2 n}$ in the second and third rows:

$$
\left[\begin{array}{llllll}
\underline{1} & 0 & 0 & 0 & 0 & 0 \\
1 & 2 & 2 & 1 & 2 & 2 \\
2 & 1 & 2 & 2 & 1 & 2 \\
0 & 0 & 0 & 0 & \underline{1} & 0 \\
0 & 0 & \underline{1} & 0 & 0 & 0 \\
0 & 0 & 0 & 0 & 0 & 1
\end{array}\right] \rightarrow\left[\begin{array}{llllll}
1 & 0 & 0 & 0 & 0 & 0 \\
0 & 2 & 0 & 1 & 0 & 0 \\
0 & 1 & 0 & 2 & 0 & 0 \\
0 & 0 & 0 & 0 & 1 & 0 \\
0 & 0 & 1 & 0 & 0 & 0 \\
0 & 0 & 0 & 0 & 0 & 1
\end{array}\right] \rightarrow\left[\begin{array}{llllll}
1 & 0 & 0 & 0 & 0 & 0 \\
0 & 1 & 0 & 0 & 0 & 0 \\
0 & 0 & 1 & 0 & 0 & 0 \\
0 & 0 & 0 & 1 & 0 & 0 \\
0 & 0 & 0 & 0 & 1 & 2 \\
0 & 0 & 0 & 0 & 2 & 1
\end{array}\right]
$$

This last matrix obviously has nonzero determinant. Notice also that $\Upsilon_{2}$ appears in the bottom right corner, and 2 is the cardinality of $S \cap B$.

Lemma 2.2 Every good set is a basis of $\boldsymbol{R}^{2 k}$.

Proof Let $S$ be a good set. Let $b$ be the cardinality of $S \cap B$. Using permutations and simple row reduction we see that

$$
\operatorname{det}(M)=s \operatorname{det}\left[\begin{array}{cc}
I & 0 \\
0 & \frac{1}{2 n} \Upsilon_{b}
\end{array}\right] \neq 0
$$

Here $s \in\{-1,1\}$ depends on the number of permutations.

Lemma 2.3 Let $\left\langle S_{1}\right\rangle$ be a good simplex. Each codimension-1 face $\left\langle S^{\prime}\right\rangle$ of $\left\langle S_{1}\right\rangle$, which is not a face of $\Delta_{0}$, is a face of one other good simplex $\left\langle S_{2}\right\rangle$. Equation 5 holds for $\left\langle S_{1}\right\rangle$ and $\left\langle S_{2}\right\rangle$.

Proof We have $S^{\prime}=S_{1}-Y_{j}$ for some $j \in\{1, \ldots, k\}$ and $Y_{j} \in\left\{A_{j}, B_{j}, C_{j}\right\}$. Without loss of generality assume $j=1$. By hypotheses $S^{\prime} \not \subset A \cup C$. Hence there is exactly one other way to complete $S^{\prime}$ to a good subset: Namely, $S_{2}=$ $S^{\prime} \cup Z_{1}$, where $Z_{1}=\left\{A_{1}, B_{1}, C_{1}\right\}-S_{1}$. Let $M_{Y}$ and $M_{Z}$ denote the matrices whose rows are the elements of $S_{1}$ and $S_{2}$ respectively. We require that $Y_{1}$ and $Z_{1}$ appear in the same rows of $M_{Y}$ and $M_{Z}$ respectively and that all other rows coincide. To verify Equation 5 for $\left\langle S_{1}\right\rangle$ and $\left\langle S_{2}\right\rangle$ it suffices to prove that $\operatorname{det}\left(M_{Y}\right) / \operatorname{det}\left(M_{Z}\right)<0$. The idea here is that this causes $Y_{1}$ and $Z_{1}$ to lie on opposite sides of the hyperplane containing $\left\langle S^{\prime}\right\rangle$. By symmetry it suffices to consider the cases $(Y, Z)=(B, C)$ and $(Y, Z)=(A, C)$. We will consider these in turn. 
Case 1 Let $b$ be the cardinality of $S \cap B$. Since $S^{\prime}=S_{1}-B_{1} \not \subset A \cup C$ we have $b \geq 2$. Using the operations of Lemma 2.2 we get the formula in Equation 7 for $\operatorname{det}\left(M_{B}\right)$. When we perform the same operations on $M_{C}$ we get the same matrix as in Equation 17 except that all the 2's in one of the rows are changed to 0 's. We can then perform one more simple row reduction, using this row, to get

$$
\operatorname{det}\left(M_{C}\right)=s \operatorname{det}\left[\begin{array}{cc}
I & 0 \\
0 & \frac{1}{2 n} \Upsilon_{b}^{(a)}
\end{array}\right]
$$

for some $a \in\{1, \ldots, b\}$. Here $\Upsilon_{b}^{(a)}$ is created from $\Upsilon_{b}$ by changing the $(a j)$ th and $(j a)$ th entries of $\Upsilon_{b}$ from 2 to 0 , for all $j \neq a$. Independent of $a$ we have

$$
\operatorname{det}\left(\Upsilon_{b}^{(a)}\right)=\operatorname{det}\left(\Upsilon_{b-1}\right) .
$$

Equations 6] give $\operatorname{det}\left(M_{B}\right) / \operatorname{det}\left(M_{C}\right)<0$.

Case 2 Suppose that $A_{1}$ and $B_{1}$ are the first two rows of $M_{A}$ and that $C_{1}$ and $B_{1}$ are the first two rows of $M_{C}$. Let $M$ be the matrix obtained by replacing the first row of $M_{A}$ (or $M_{C}$ ) by $A_{1}+C_{1}$. We have $\operatorname{det}(M)=\operatorname{det}\left(M_{A}\right)+\operatorname{det}\left(M_{C}\right)$. The first row of $M$ is $(1,0, \ldots, 0,1,0, \ldots, 0)$. Using this row for row-reduction we can make all other rows have zeros in the 1 st and $(k+1)$ st positions. The last $2 k-2$ rows of $M$ are linearly independent by Lemma 2.2. Therefore we can perform a series of row reductions to change the remaining entries of the second row of $M$ to 0 s. Hence $\operatorname{det}(M)=0$ and $\operatorname{det}\left(M_{A}\right) / \operatorname{det}\left(M_{C}\right)=-1$.

Remark To be sure we checked all the calculations entailed by the preceding lemma by computer for the cases $n=3,5,7,9,11,13$.

Corollary 2.4 $\Delta_{0}$ is the union of the good simplices.

Proof Let $\Delta^{\prime}$ be the union of the good simplices. $\Delta^{\prime}$ is closed subset of $\Delta_{0}$. If $\Delta^{\prime} \neq \Delta_{0}$ then some codimension one subset of $\partial \Delta^{\prime}$ separates the nonempty $\operatorname{int}\left(\Delta_{0}-\Delta^{\prime}\right)$ from the nonempty $\operatorname{int}\left(\Delta^{\prime}\right)$. Hence there is a good simplex $\left\langle S_{1}\right\rangle$, a codimension 1 face $\left\langle S^{\prime}\right\rangle$ of $\left\langle S_{1}\right\rangle$, and a point $x \in \operatorname{int}\left(\Delta_{0}\right) \cap \operatorname{int}\left(\left\langle S^{\prime}\right\rangle\right) \cap \partial \Delta^{\prime}$. Note that $\left\langle S^{\prime}\right\rangle$ is not a face of $\Delta_{0}$. By Lemma 2.3 there is a good simplex $\left\langle S_{2}\right\rangle$ which also has $\left\langle S^{\prime}\right\rangle$ as a face, and $x \in \operatorname{int}\left(\left\langle S_{1}\right\rangle \cup\left\langle S_{2}\right\rangle\right) \subset \operatorname{int}\left(\Delta^{\prime}\right)$. This is a contradiction.

Corollary 2.5 Equation 5 is true for all good simplices $\left\langle S_{1}\right\rangle$ and $\left\langle S_{2}\right\rangle$ provided that $\left\langle S_{1} \cap S_{2}\right\rangle$ has codimension less than 3 . 
Proof Lemma 2.3 takes care of the codimension 1 case. Let $F=\left\langle S_{1} \cap S_{2}\right\rangle$ have codimension 2. We will treat the case when $F$ is not a face of $\Delta_{0}$, the other case being very similar.

There are either 3 or 4 ways to complete $S_{1} \cap S_{2}$ to a good set. From Lemma2.3 the corresponding good simplices just wind around $F$ in a cyclic fashion. That is, there is a cyclic ordering to the simplices, such that consecutive simplices are as in Lemma 2.3. The simplices are prevented from winding more than once around $F$ by the fact that the total dihedral angle around $F$ is less than $4 \times \pi=4 \pi$. The topological situation just described implies that $\left\langle S_{1}\right\rangle \cap\left\langle S_{2}\right\rangle=$ $F$.

Corollary 2.6 Equation 5 holds for every pair of good simplices.

Proof Let $\Delta=\Delta_{0}$. Let $\widehat{\Delta}$ be the abstract simplicial complex obtained by gluing together all the good simplices along the convex hulls of their common vertices. We have a tautological map $I: \widehat{\Delta} \rightarrow \Delta$ which maps each abstract version of a good simplex to its realization as a subset of $\Delta$. It suffices to prove that $I$ is a bijection.

Let $\widehat{\Delta}_{k}$ denote the interior of the complement of the codimension- $k$ skeleton of $\widehat{\Delta}$. Corollary 2.5 implies that $I$ is a local isometry on $\widehat{\Delta}_{3}$. The point here is that we just need to look at the links of interior simplices of codimension 1 and 2 , and this is what we have done.

$I$ maps the codimension- 3 skeleton of $\widehat{\Delta}$ onto the set of codimension- 3 faces of the good simplices. Since $I$ is onto (by Corollary 2.4) the set $\Delta_{3}=\operatorname{int}\left(I\left(\widehat{\Delta}_{3}\right)\right.$ ) is obtained from $\operatorname{int}(\Delta)$ by deleting the codimension- 3 faces. Hence $\Delta_{3}$ is open, simply connected and dense. We can find a local isometry $J$, defined on an open subset of $\Delta_{3}$, which is the inverse of $I$ where defined. Since $\Delta_{3}$ is open and simply connected, $J$ extends by analytic continuation to a local isometry on $\Delta_{3}$. Since $\Delta_{3}$ is dense, $J$ extends to all of $\Delta$.

Since $\widehat{\Delta}_{3}$ and $\Delta_{3}$ are both simply connected it follows from analytic continuation that the local isometries $I \circ J$ and $J \circ I$ are the identity on $\Delta_{3}$ and $\widehat{\Delta}_{3}$ respectively. By continuity, they are the identity on $\Delta$ and $\widehat{\Delta}$ respectively. Hence $I$ is a bijection.

\section{$2.3 \quad 3$-dimensional example}

We illustrate our construction by working out the 3 dimensional case more explicitly. To get a 3-dimensional picture we use the projection

$$
V \rightarrow(V \cdot(1,1,-1,-1), V \cdot(1,-1,1,-1), V \cdot(1,-1,-1,1))
$$


Using this projection we have

$$
A_{1}:\left[\begin{array}{l}
1 \\
1 \\
1
\end{array}\right] \quad A_{2}:\left[\begin{array}{c}
1 \\
-1 \\
-1
\end{array}\right] \quad B_{1}:\left[\begin{array}{c}
0 \\
-\frac{1}{3} \\
0
\end{array}\right] \quad B_{2}:\left[\begin{array}{l}
0 \\
\frac{1}{3} \\
0
\end{array}\right] \quad C_{1}:\left[\begin{array}{c}
-1 \\
1 \\
-1
\end{array}\right] \quad C_{2}:\left[\begin{array}{c}
-1 \\
-1 \\
1
\end{array}\right]
$$

Figure 2.1 shows a projection to the $x y$ plane. The two tetrahedra on the right are supposed to fit inside the one on the left, as indicated by the labels.
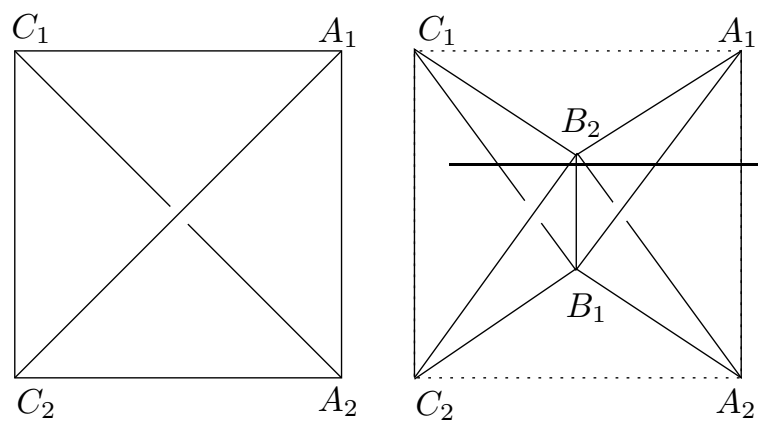

Figure 2.1

The 6 tetrahedra which partition $\Omega$ are glued together along common faces, in the following cyclic pattern.

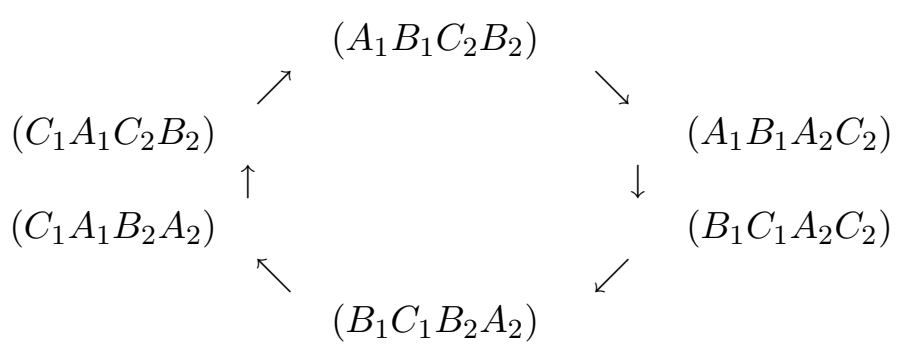

The action of $\sigma$ translates this cycle of tetrahedra one third of the way around. A study of this pattern led us to the general case.

\section{Theorem 1.1}

\subsection{The modular tiling}

We use the disk model of $\boldsymbol{H}^{2}$. By slight abuse of terminology, we still say that $P S L_{2}(\boldsymbol{Z})$ acts on this model. Technically $P S L_{2}(\boldsymbol{Z})$ acts on the upper half plane model and a conjugate of $P S L_{2}(\boldsymbol{Z})$ acts on the disk model. 
$\boldsymbol{H}^{2}$ has a canonical (and familiar) tiling $T$ by ideal triangles which is invariant under the action of $P S L_{2}(\boldsymbol{Z})$. We define $T$ by saying that it is the orbit of an ideal triangle under the group generated by reflections in its own sides. See [5. page 298] for a beautiful picture. We call $T$ the modular tiling. Let $V T$, $E T$, and $F T$ respectively denote the set of ideal vertices, geodesic edges, and ideal triangles of $T$. We say that two elements of ET are touching if they are identical or share a common endpoint. We say that two elements of $F T$ are touching if they are identical or share a common edge.

$T$ defines an exhaustion of $\boldsymbol{H}^{2}$ by ideal polygons. Let $t_{+} \in F T$ be some distinguished ideal triangle. Let $T_{0}=\left\{t_{+}\right\}$and inductively define $T_{m+1}$ to be those ideal triangles of $T$ which are touching ideal triangles of $T_{m}$. Then $T_{m}$ is an ideal polygon with $3 \times 2^{m}$ sides. The combinatorial distance between $\tau_{1}, \tau_{2} \in F T$ is the number of edges in ET crossed by the geodesic segment which connects the centers of $\tau_{1}$ and $\tau_{2}$. The triangles in $T_{m+1}-T_{m}$ are those which are have combinatorial distance $m$ from $t_{+}$.

Each $e \in E T$ bounds a unique open halfspace $h_{e}$ which is disjoint from the interior of $t_{+}$. Given $x \in S^{1}$ we write $e \mid x$ if $x$ is an accumulation point of $h_{e}$. The set of $x$ such that $e \mid x$ is one of the two closed arcs on $S^{1}$ determined by the endpoints of $e$. Each $x \in S^{1}-V T$ defines a unique maximal sequence $\left\{e_{m}\right\}$ of edges such that $e_{m} \mid x$ for all $x$ and $h_{m+1} \subset h_{m}$ for all $m$. We call $\left\{e_{m}\right\}$ the nesting sequence for $x$.

Using the disk model of $\boldsymbol{H}^{2}$ we can put a metric on $\boldsymbol{H}^{2} \cup S^{1}$ which makes it isometric to a closed Euclidean disk. The next result refers to this metric.

Lemma 3.1 For any $\eta>0$ there is a $\delta>0$ such that: If $x_{1}, x_{2} \in S^{1}$ are less than $\delta$ apart then there are touching edges $e_{1}, e_{2} \in E T$ such that $e_{1} \mid x_{1}$ and $e_{2} \mid x_{2}$.

Proof There is some $m$ such that all edges of $\partial T_{m}$, which is an ideal polygon, have diameter less than $\eta$. We take $\delta$ to be the minimim distance on $S^{1}$ between vertices of this ideal polygon.

\subsection{Modular pattern basics}

Throughout this chapter $\Gamma$ will denote a modular pattern of geodesics.

Lemma 3.2 Let $\Gamma$ be a modular pattern of geodesics. 
(1) The endpoint of a geodesic of $\Gamma$ belongs to $S^{1}-V T$.

(2) Two geodesics of $\Gamma$ cannot share an endpoint.

(3) Each $e \in E T$ intersects only finitely many geodesics of $\Gamma$.

Proof Let $G$ be a finite index torsion-free subgroup of $P S L_{2}(\boldsymbol{Z})$. Then $T / G$ is a tiling of the finite area surface $\Sigma=\boldsymbol{H}^{2} / G$ by ideal triangles. Each individual edge of $T$ maps injectively onto an edge of $T / G$. The quotient $\Gamma / G$ is a finite union of closed geodesics on $\Sigma$. To prove Item 1 , suppose a geodesic $\gamma \in \Gamma$ has an endpoint $v \in V T$. Then $\gamma / G$ exits every compact subset of $\Sigma$ as it approaches the cusp point on $\Sigma$ corresponding to $v$. Closed geodesics have finite length and hence don't do this. Item 2 follows from the general fact, applied to $\gamma_{1} / G$ and $\gamma_{2} / G$, that two closed geodesics on a complete hyperbolic surface cannot have lifts which share exactly one endpoint. To prove Item 3 , note that the set $(e / G) \cap(\Gamma / G)$ is finite by compactness. Since the map $e \rightarrow e / G$ is injective the set $e \cap \Gamma$ is also finite.

Item 2 above shows that the relation $\sim$ defined in section 1 is an equivalence relation: The transitivity condition is vacuously satisfied.

Corollary 3.3 There is some $m$ such that: If $\tau_{1}, \tau_{2} \in F T$ have combinatorial distance at least $m$ then at most one geodesic of $\Gamma$ intersects both $\tau_{1}$ and $\tau_{2}$.

Proof By symmetry we can take $\tau_{1}=t_{+}$, the distinguished triangle. If $\left\{t_{m}\right\}$ was a sequence of counterexamples to this lemma then there would be two geodesics of $\Gamma$ intersecting both $t_{+}$and $t_{m}$ for all $m$. Taking a subsequence we can assume that $t_{m}$ converges to some $x \in S^{1}$. There are only finitely many geodesics which intersect $t_{+}$. Hence, taking another subsequence, we get the same two geodesics intersecting $t_{m}$ for all $m$. But then $x$ would be an endpoint to both geodesics, contradicting Item 2 of Lemma 3.2 .

\subsection{Simplicial correspondences}

We continue with the notation established above. For each $e \in E T$ let $\Gamma_{e}$ denote the set with the following description. An object is an element of $\Gamma_{e}$ iff it is either an endpoint of $e$ or a geodesic of $\Gamma$ which crosses $e$. The cardinality of $\Gamma_{e}$ is $|\Gamma|+1$, where $|\Gamma|$ is as in Theorem 1.1. This quantity is finite by Lemma 3.2 and independent of $e$ by symmetry. As in the statement of Theorem 1.1 we let $n=|\Gamma|$. 
Recall from section 2.1 that $S^{n}=\Delta_{+} \cup \Delta_{-}$. We equip $S^{n}$ with the piecewise Euclidean metric inherited from $\Delta_{+}$and $\Delta_{-}$. As in section 1.1, a simplex of $S^{n}$ is defined to be a sub-simplex of either $\Delta_{+}$or $\Delta_{-}$. If $\Delta$ is an $n$-simplex of $S^{n}$ there is a bijective map from $\Gamma_{e}$ to $V \Delta$, the vertex set of $\Delta$, because the two sets have the same cardinality. Compare our table at the end of section 1.

To each $e \in E T$ we assign a pair

$$
\Psi(e)=\left(\Delta_{e}, \phi_{e}\right),
$$

where $\Delta_{e}$ is an $n$-dimensional simplex of $S^{n}$ and $\phi_{e}: \Gamma_{e} \rightarrow V \Delta_{e}$ is a bijection. When the map $\phi_{e}$ is not immediately under discussion we will sometimes abuse notation and write $\Delta_{e}=\Psi(e)$.

We say that $\Psi$ is a simplicial correspondence for $\Gamma$ if it satisfies the following 3 properties:

Property 1 For any $\epsilon>0$ there are only finitely many simplices in the image of $\Psi$ which have diameter greater than $\epsilon$.

Property 2 Given $e_{1}$ and $e_{2}$ in $E T$ we let $\left(\Delta_{j}, \phi_{j}\right)=\Psi\left(e_{j}\right)$. Suppose $v_{j}$ is a vertex of $\Delta_{j}$ for $j=1,2$. Then $v_{1}=v_{2}$ iff $\phi_{1}^{-1}\left(v_{1}\right)=\phi_{2}^{-1}\left(v_{2}\right)$. So, each vertex in the grand union $\Psi(E T)$ is labelled by a unique element of $\Gamma \cap V T$. Compare the table at the end of section 1 .

Property 3 Let $e_{1}, e_{2}, \Delta_{1}$ and $\Delta_{2}$ be as in Property 2 . Let $h_{j}=h_{e_{j}}$ for $j=1,2$, as defined in section 3.1. We require that $\operatorname{int}\left(\Delta_{1}\right) \subset \operatorname{int}\left(\Delta_{2}\right)$ iff $h_{1} \subset h_{1}$ and $\operatorname{int}\left(\Delta_{1}\right) \cap \operatorname{int}\left(\Delta_{2}\right)=\emptyset$ iff $h_{1} \cap h_{2}=\emptyset$. We also require that $\partial \Delta_{1} \cap \partial \Delta_{2}$ is the convex hull of their common vertices. So, the simplices have the same nesting properties as the open half spaces.

We will construct $\Psi$ in section 3.6. First we want to explore the consequences of its existence.

\subsection{Embedding the quotient}

In this section we use $\Psi$ to define an embedding $i: Q_{\Gamma} \rightarrow S^{n}$. Let $x \in S^{1}$. Referring to the notation of section 3.1, there is a sequence $\left\{e_{m}\right\}_{m=1}^{\infty} \in E T$ such that $e_{m} \mid x$ for all $m$. (This is true even if $x \in V T$, but there is not a unique maximal sequence in this case.) Let $\Delta_{m}=\Psi\left(e_{m}\right)$ and

$$
\Psi_{\infty}(x)=\bigcap_{n=1}^{\infty} \Delta_{m}
$$


Lemma 3.4 $\Psi_{\infty}$ is well defined.

Proof If $x \in V T$ then $x$ is an endpoint of $e_{m}$ for all $m$. Hence $\phi_{m}(x) \in V \Delta_{m}$ for all $m$. Hence $\Psi_{\infty}(x)=\phi_{m}(x)$, independent of $m$ and the choice of $\left\{e_{m}\right\}$. If $x \in S^{1}-V T$ then any sequence used to define $\Psi_{\infty}(x)$ is contained in the nesting sequence for $x$. The intersection in Equation [1] is nested, by Property 3 , and is a single point, by Property 1 .

Lemma 3.5 $\Psi_{\infty}$ identifies points on $S^{1}$ if and only if they are equivalent.

Proof If $x, x^{\prime} \in S^{1}$ are endpoints of a geodesic $\gamma$ in $\Gamma$ then by Lemma 3.2 we have $x, x^{\prime} \in S^{1}-V T$. Let $\left\{e_{m}\right\}$ and $\left\{e_{m}^{\prime}\right\}$ be the nesting sequences for $x$ and $x^{\prime}$ respectively. Then $\gamma$ crosses $e_{m}$ and $e_{m}^{\prime}$ for all $m$ and $\Delta_{m}$ and $\Delta_{m}^{\prime}$ share a vertex for all $m$, by Property 2 . Hence $\Psi_{\infty}(x)=\Psi_{\infty}\left(x^{\prime}\right)$.

If $x, x^{\prime} \in S^{1}$ are inequivalent then there are edges $e, e^{\prime} \in E T$ such that

(1) $h_{e} \cap h_{e^{\prime}}=\emptyset$.

(2) $e$ and $e^{\prime}$ have no vertices in common.

(3) $e \mid x$ and $e \mid x^{\prime}$.

(4) No geodesic of $\Gamma$ crosses both $e$ and $e^{\prime}$.

If this was false then we could take a limit of a sequence of counterexamples and produce a geodesic of $\Gamma$ whose endpoints were $x$ and $x^{\prime}$.

Now $\Gamma_{e} \cap \Gamma_{e^{\prime}}=\emptyset$ by Items 2 and 4 . By Property 2 , the simplices $\Delta_{e}$ and $\Delta_{e^{\prime}}$ corresponding to $e$ and $e^{\prime}$ have no vertices in common. Hence $\Delta_{e} \cap \Delta_{e^{\prime}}=\emptyset$ by Property 3 and Item 1 . From the definition of $\Psi$ and Item 3 we have $\Psi_{\infty}(x) \in \Delta_{e}$ and $\Psi_{\infty}\left(x^{\prime}\right) \in \Delta_{e^{\prime}}$. Hence $\Psi_{\infty}(x) \neq \Psi_{\infty}\left(x^{\prime}\right)$.

Lemma 3.6 $\Psi_{\infty}$ is continuous.

Proof Let $\|\cdot\|$ denote the diameter in the piecewise Euclidean metric on $S^{n}$ and also the Euclidean diameter on $\boldsymbol{H}^{2} \cup S^{1}$. Let $\epsilon>0$ be given. By Property 1 there is some $\eta>0$ such that: If $e \in E T$ satisfies $\|e\|<\eta$ then $\left\|\Delta_{e}\right\|<\epsilon / 2$. Here $\Delta_{e}=\Psi(e)$. Let $\delta$ be as in Lemma 3.1. If $\operatorname{dist}\left(x_{1}, x_{2}\right)<\delta$ then there are touching $e_{1}, e_{2} \in E T$ such that $e_{j} \mid x_{j}$ and $\left\|e_{j}\right\|<\eta$ for $j=$ 1,2. But then $\Psi_{\infty}\left(x_{1}\right)$ and $\Psi_{\infty}\left(x_{2}\right)$ are contained in simplices $\Delta_{1}$ and $\Delta_{2}$ which by Property 2 share at least one vertex. Moreover $\left\|\Delta_{j}\right\|<\epsilon / 2$. Hence $\operatorname{dist}\left(\Psi_{\infty}\left(x_{1}\right)-\Psi_{\infty}\left(x_{2}\right)\right)<\epsilon$. 
Define

$$
\Lambda=\Psi_{\infty}\left(S^{1}\right) .
$$

Combining the last two results we see that $\Psi_{\infty}$ factors through a continuous bijection $i: Q_{\Gamma} \rightarrow \Lambda$. A continuous bijection from a compact space to a Hausdorff space is a homeomorphism. Thus $i$ is a homeomorphism. This is our embedding from Theorem 1.1

Here we give a useful characterization of $\Lambda$.

\section{Lemma 3.7}

$$
\Lambda=\bigcap_{m=0}^{\infty} \Lambda_{m} \quad \text { where } \quad \Lambda_{m}=\bigcup_{e \in \partial T_{m}} \Delta_{e} .
$$

Proof We have $\Lambda \subset \Lambda_{m}$ by Property 3 and the definition of $\Psi$. Any $y \in \cap \Lambda_{m}$ is contained in an infinite nested sequence $\left\{\Delta_{m}\right\}$ of simplices. By Property 3 the corresponding sequence $\left\{e_{m}\right\}$ is such that $e_{m} \mid x$ for some $x \in S^{1}$ and for all $m$. Thus $\Psi_{\infty}(x)=y$. Hence $\bigcap \Lambda_{m} \subset \Lambda$.

Remark As above we set $\Psi(e)=\left(\Delta_{e}, \phi_{e}\right)$. By Property 2 all the local maps $\left\{\phi_{e} \mid e \in E T\right\}$ piece together to give a global bijection:

$$
\left[\Gamma \cup V T=\bigcup_{e \in E T} \Gamma_{e}\right] \stackrel{\phi}{\longleftrightarrow} \quad\left[V \Psi=\bigcup_{e \in E T} V \Delta_{e}\right]
$$

If $x \in V T$ then $\Psi_{\infty}(x)=\phi(x)$. If $x$ is an endpoint of a geodesic $\gamma$ of $\Gamma$ then $\Psi_{\infty}(x)=\phi(\gamma)$. Therefore $\Lambda$ is the closure of $V \Psi$.

\subsection{Block networks}

Let $\Omega_{+}$be the modular block from section 2 . We will only use modular blocks in $S^{n}$ which have the following definition: Let $\Delta \subset S^{n}$ be a simplex. Let $A: \Delta_{+} \rightarrow \Delta$ be an affine isomorphism. Our new modular block is $A\left(\Omega_{+}\right)$. The outer terminal is $\partial \Delta$. Every two modular blocks in $S^{n}$ (that we use) are affinely equivalent. $A$ maps the canonical triangulation of $\Omega_{+}$to a canonical triangulation of $A\left(\Omega_{+}\right)$. Given two modular blocks $\Omega_{1}, \Omega_{2} \subset S^{n}$ we let $\operatorname{Map}\left(\Omega_{1}, \Omega_{2}\right)$ be the set of triangulation-respecting PL maps from $\Omega_{1}$ to $\Omega_{2}$.

Given $\tau \in F T$ we define

$$
\Gamma_{\tau}=\bigcup_{e \in \partial \tau} \Gamma_{e}
$$


For each edge $e \in E T$, the set $\Gamma_{e}$ has $2 k$ elements. Each $\Gamma_{e}$ shares $k$ of its elements with another $\Gamma_{e^{\prime}}$. Hence $\Gamma_{\tau}$ has $3 k$ elements. An $n$-dimensional modular block $\Omega$ also has $3 k$ vertices. Let $g \in P S L_{2}(\boldsymbol{Z})$ be the element which cycles the 3 edges of $\tau$ in counterclockwise order. Let $\sigma$ be the 3 -fold PL symmetry of $\Omega$. We say that a $\tau$-labelling of $\Omega$ is a bijection $\phi: \Gamma_{\tau} \rightarrow V \Omega$ which satisfies $\phi \circ g=\sigma \circ \phi$. Here $V \Omega$ is the vertex set of $\Omega$.

Lemma 3.8 If $\Omega_{1}$ and $\Omega_{2}$ are two $\tau$-labelled modular blocks then there is a unique element of $\operatorname{Map}\left(\Omega_{1}, \Omega_{2}\right)$ which carries the one labelling to the other. This element is affine if it matches up the outer terminals.

Proof Composing with affine maps we reduce to the case $\Omega_{1}=\Omega_{2}=\Omega_{+}$. Note first that $\operatorname{Map}\left(\Omega_{+}, \Omega_{+}\right)$is quite large: Any permutation $\pi$ of the the $k-$ element set $A=\left\{A_{j}\right\}_{j=1}^{k} \subset V \Omega_{+}$extends to an element $I_{\pi} \in \operatorname{Map}\left(\Omega_{+}, \Omega_{+}\right)$ which is an isometry. The map $I$ commutes with $\sigma$, the 3 -fold symmetry of $\Omega_{+}$, and (hence) permutes the indices of the vectors in $B$ and $C$ in the same way it permutes the indices of the vectors in $A$.

Let $\phi$ and $\phi^{\prime}$ be two $\tau$-labellings of $\Omega_{+}$. Let $e_{0}, e_{1}, e_{2}$ be the three edges of $\tau$ and let $\Delta_{0}, \Delta_{1}, \Delta_{2}$ be the simplices associated to $\Omega_{+}$. Let $\Gamma_{j}=\Gamma_{e_{j}}$. Let $S=\Gamma_{0} \cap \Gamma_{1}$. Composing $\phi$ with $\sigma^{a}$ for some $a \in\{0,1,2\}$ we can assume that $\phi\left(\Gamma_{j}\right)=V \Delta_{j}=\phi^{\prime}\left(\Gamma_{j}\right)$ for $j=0,1,2$. By symmetry $\phi$ and $\phi^{\prime}$ are determined by their action on $S$. Also $\phi(S)=\phi^{\prime}(S)$. Hence there is some permutation $\pi$ of $A=V \Delta_{0} \cap V \Delta_{1}$ such that $\left.\phi\right|_{S}=\left.\pi \circ \phi^{\prime}\right|_{S}$. Referring to the extension of $\pi$ discussed above, we have $\phi=I_{\pi} \circ \phi^{\prime}$.

We say that a block network is an assignment $\tau \rightarrow \Omega[\tau]$, for each $\tau \in F T$. Here $\Omega[\tau]$ is a $\tau$-labelled modular block. We require that $\Omega\left[t_{+}\right]=\Omega_{+}$and

(1) $\Omega\left[\tau_{1}\right]$ and $\Omega\left[\tau_{2}\right]$ have disjoint interiors for all $\tau_{1} \neq \tau_{2}$.

(2) $\Omega\left[\tau_{1}\right]$ and $\Omega\left[\tau_{2}\right]$ share a common terminal if $\tau_{1}$ and $\tau_{2}$ share an edge.

(3) $\Omega\left[\tau_{1}\right]$ and $\Omega\left[\tau_{2}\right]$ share a common vertex $v$ if and only if the $\tau_{1}$ label of $v$ coincides with the $\tau_{2}$ label of $v$.

Lemma 3.9 There exists a block network for $\Gamma$.

Proof We choose an enumeration $t_{+}=t_{0}, t_{1}, t_{2}, \ldots$ of the ideal triangles of $F T$ with the following property: For any $w \geq 1$, each $t_{w}$ shares an edge $e$ with some $t_{v}$ for some $v<w$. We will define $\Omega_{w}=\Omega\left[t_{w}\right]$ inductively. We define $\Omega_{0}=\Omega_{+}$as we must. We choose some $t_{0}$-labelling for $\Omega_{0}$. Note that $S^{n}-\Omega_{0}$ 
consists of 3 disjoint open simplices: $\operatorname{int}\left(\Delta_{-}\right)$and $\operatorname{int}\left(\Delta_{1}\right)$ and $\operatorname{int}\left(\Delta_{2}\right)$. We call these simplices holes. Each edge of $t_{0}$ corresponds to a hole.

Suppose that $\Omega_{0}, \ldots, \Omega_{w-1}$ have been defined, and each edge of the polygon $P_{w}=\partial\left(t_{0} \cup \ldots \cup t_{w-1}\right)$ is associated to an open simplex-i.e. a hole - of $S^{n}-$ $\bigcup_{j=1}^{w-1} \Omega_{j}$, There is some edge $e$ of $P_{w}$ which bounds $t_{w}$ and some $v<w$ such that $t_{v}$ and $t_{w}$ share $e$ as an edge. Let $\Delta$ be the simplex which is the closure of the corresponding hole in $S^{n}$. Note that $V \Delta$ is already labelled by elements of $\Gamma_{e}$. The labelling comes from the $t_{v}$-labelling of $\Omega_{v}$, which has $\partial \Delta$ a terminal. First we choose a $t_{w}$-labelling of $\Omega_{0}$ such that the outer terminal $\partial \Delta_{+}$is labelled by elements of $\Gamma_{e}$. Next we choose the unique affine isomorphism $A: \Delta_{+} \rightarrow \Delta$ which matches the $t_{w}$-labelling of $\Delta_{+}$with the $t_{v}$-labelling of $\Delta$. We define $\Omega_{w}=A\left(\Omega_{0}\right)$. We use $A$ to give $\Omega_{w}$ a $t_{w}$-labelling. The hole $\Delta$ has been plugged up but the two inner terminals of $\Omega_{w}$ bound two new holes.

Our construction only identifies vertices when they correspond to the same object of $\Gamma \cup V T$. No vertices are identified by accident because of the way the blocks are nested. These same nesting properties show that all the blocks have disjoint interiors. Thus we have constructed a block network.

Remark The axioms for block networks imply that any block network for $\Gamma$ can be constructed by our inductive process. Once we determine the $t_{0}-$ labelling the rest of the construction is forced. Different $t_{0}$-labellings produce geometrically identical networks, but with the labels permuted.

\subsection{Putting it together}

Let $\Omega[*]$ be our block network. We define $\Psi(e)=\left(\Delta_{e}, \phi_{e}\right)$, where $\Delta_{e}$ is the relevant simplex of $\Omega[\tau]$ and $\phi_{e}$ is the restriction of the $\tau$ labelling to $\Gamma_{e}$. Here $\tau$ is one of the two triangles which has $e$ as an edge. From the block network axioms, either choice of $\tau$ gives the same map.

Lemma 3.10 $\Psi$ is a simplicial correspondence.

Proof Properties 2 and 3 are immediate from our construction. It suffices to check property 1 . Given two edges $e, e^{\prime} \in E T$ we write $e \rightarrow_{1} e^{\prime}$ if $h_{e} \subset h_{e^{\prime}}$ and if $e$ and $e^{\prime}$ bound a common ideal triangle of $T$. We inductively define $e \rightarrow_{(m+1)} e^{\prime}$ iff $e \rightarrow_{m} e^{\prime \prime}$ and $e^{\prime \prime} \rightarrow_{1} e^{\prime}$. We let $\Delta_{e}=\Psi(e)$ and $\Delta_{e^{\prime}}=\Psi\left(e^{\prime}\right)$. By Corollary 3.3. Property 2, and Property 3, there is some $m$ such that: If $e \rightarrow_{m} e^{\prime}$ then $\partial \Delta_{e} \cap \partial \Delta_{e^{\prime}}$ is at most a single point. We fix $m$. 
Let $\mathcal{S}$ denote the set of pairs of simplices of the form $\left(\Delta_{e}, \Delta_{e^{\prime}}\right)$, where $e \rightarrow_{m} e^{\prime}$. We say that two pairs $\left(\Delta_{1}, \Delta_{1}^{\prime}\right)$ and $\left(\Delta_{2}, \Delta_{2}^{\prime}\right)$ in $\mathcal{S}$ are equivalent if there is an affine map which carries one pair to the other. Modulo $P S L_{2}(\boldsymbol{Z})$ there are only finitely many pairs $\left(e, e^{\prime}\right)$ with $e \rightarrow_{m} e^{\prime}$. Thus, by the affine naturality in our construction, $\mathcal{S}$ contains finitely many equivalence classes.

Let $\Delta=\Delta_{+}$, the standard simplex. For each equivalence class in $\mathcal{S}$ we define a model pair $\left(\Delta, \Delta^{\prime}\right)$, affine equivalent to any member of the equivalence class. If Property 1 fails we can find a nested sequence $\Delta_{1} \supset \Delta_{2} \supset \Delta_{3} \ldots$ such that $\cap \Delta_{j}$ is more than a single point. Such a nested sequence exists by Property 3 . By taking an evenly spaced subsequence we can assume that $\left(\Delta_{j}, \Delta_{j+1}\right)$ is a member of $\mathcal{S}$ for all $j$. At least one model pair $\left(\Delta, \Delta^{\prime}\right)$ is represented infinitely often.

Let $\lambda_{j}^{\prime}$ be the longest edge of $\Delta_{j+1}$. Let $\lambda_{j}$ be the longer line segment obtained by intersecting $\Delta_{j}$ with the line containing $\lambda_{j}^{\prime}$. Since $\cap \Delta_{j}$ does not shrink to a point, length $\left(\lambda_{j}^{\prime}\right) \nrightarrow 0$. Since $\Delta_{j}$ and $\Delta_{j+1}$ converge to each other as $j \rightarrow \infty$, we have length $\left(\lambda_{j}^{\prime}\right) \rightarrow$ length $\left(\lambda_{j}\right)$. For an infinite collection of indices $j$ there is an affine map $T_{j}$ which takes the pair $\left(\Delta_{j}, \Delta_{j+1}\right)$ to the model pair $\left(\Delta, \Delta^{\prime}\right)$. Let $\delta_{j}=T_{j}\left(\lambda_{j}\right)$ and $\delta_{j}^{\prime}=T_{j}\left(\lambda_{j}^{\prime}\right)$. An affine map respects ratios of distances on lines. Hence length $\left(\delta_{j}^{\prime}\right) \rightarrow \operatorname{length}\left(\delta_{j}\right)$ and $\delta_{j}^{\prime}$ is an edge of $\Delta^{\prime}$ for all $j$. Everything takes place on the same model so the set of possible pairs $\left(\delta_{j}^{\prime}, \delta_{j}\right)$ is finite. Hence $\delta_{j}=\delta_{j}^{\prime}$ for large $j$. Hence $\partial \Delta$ and $\partial \Delta^{\prime}$ have two distinct points in common, contradicting the choice of $m$.

The work in section 3.4 gives us our embedding. Now we construct the representation from Theorem 1.1. Let $g \in P S L_{2}(\boldsymbol{Z})$. Let $\tau$ be an ideal triangle of $T$. Let $\tau^{\prime}=g(\tau)$. Let $\phi: \Gamma_{\tau} \rightarrow \Omega[\tau]$ be the $\tau$-labelling of $\Omega[\tau]$. Let $\phi^{\prime}: \Gamma_{\tau^{\prime}} \rightarrow \Omega\left[\tau^{\prime}\right]$ be the $\tau^{\prime}$-labelling of $\Omega\left[\tau^{\prime}\right]$. By Lemma 3.8 there is a unique $\rho(g, \tau) \in \operatorname{Map}\left(\Omega[\tau], \Omega\left[\tau^{\prime}\right]\right)$ such that $\rho(g, \tau) \circ \phi=\phi^{\prime}$. In other words $\rho(g, \tau)$ maps the vertex of $\Omega[\tau]$ labelled by the object $x$ to the vertex of $\Omega\left[\tau^{\prime}\right]$ labelled by the object $x^{\prime}=g(x)$. This intertwining property implies that $\rho\left(g, \tau_{1}\right)$ and $\rho\left(g, \tau_{2}\right)$ agree on any common vertices. Since $\Omega\left[\tau_{1}\right] \cap \Omega\left[\tau_{2}\right]$ is contained in a single simplex, on which both our maps are affine, we see that $\rho\left(g, \tau_{1}\right)=\rho\left(g, \tau_{2}\right)$ on $\Omega\left[\tau_{1}\right] \cap \Omega\left[\tau_{2}\right]$. Letting $\widehat{\Omega}=\bigcup \Omega[\tau]$ we see that the $\rho(g, \tau)$ maps piece together to give a continuous map $\rho(g): \widehat{\Omega} \rightarrow \widehat{\Omega}$. The intertwining property gives

$$
\rho\left(g_{1} g_{2}\right)=\rho\left(g_{1}\right) \rho\left(g_{2}\right) \text {. }
$$

We now show that $\rho(g)$ extends to an element of $\operatorname{PL}\left(S^{n}\right)$. There is some $m$ such that $t_{+} \subset g\left(T_{m}\right)$. If $e$ is an edge of $\tau_{1} \in F T-T_{m}$ then $h_{e} \cap T_{m}=\emptyset$. Therefore $\tau_{1} \notin g\left(h_{e}\right)$. Therefore $g\left(h_{e}\right)=h_{g(e)}$. Therefore $\rho\left(g, \tau_{1}\right)$ identifies 
the outer terminals of the two blocks and by Lemma 3.8 is affine. If $\tau_{2}$ is an ideal triangle touching $\tau_{1}$ and contained in $h_{e}$ then $\Omega\left[\tau_{1}\right]$ and $\Omega\left[\tau_{2}\right]$ intersect along the outer terminal $\partial \Delta$ of $\Omega\left[\tau_{1}\right]$. Since two affine maps are determined by their action on a simplex we see that $\rho\left(g, \tau_{1}\right)$ and $\rho\left(g, \tau_{2}\right)$ are restrictions of the same affine map. Repeating this argument with $\tau_{2}$ replacing $\tau_{1}$, etc., we see inductively that $\rho(g)$ is affine on all blocks contained in $\Delta$. We extend $\rho(g)$ by making it affine on all of $\Delta$. Since there are only finitely many edges of $T_{m+1}$ we see that the extension of $\rho(g)$ is PL on the set $\Lambda_{m+1}$ defined in Lemma 3.7 There are only finitely many blocks not contained in $\Lambda_{m+1}$ and $\rho(g)$ is PL on each one. In summary, $\rho(g)$ is a PL map. Equation [15 shows that $\rho(g)$ has the inverse $\rho\left(g^{-1}\right)$ which is also PL. Hence $\rho(g) \in \operatorname{PL}\left(S^{n}\right)$.

Equation [15] says that the map $g \rightarrow \rho(g)$ is a homomorphism. Every $\rho(g)$ acts

nontrivially on some block. Hence $\rho$ is a monomorphism. $\Lambda$ is the closure of the block network vertices. Hence $H=\rho\left(P S L_{2}(\boldsymbol{Z})\right)$ preserves $\Lambda$. From the remark at the end of section 3.4, the map $\Psi$ conjugates the minimal action of $P S L_{2}(\boldsymbol{Z})$ on $\partial \boldsymbol{H}^{2}$ to a minimal action of $H$ on $\Lambda$. The triangulations of all the blocks piece together to give a partition of $S^{n}-\Lambda$ by punctured simplices. Corollary 3.2 and the local finiteness of the modular tiling imply that our partition by punctured simplices is locally finite. $H$ permutes this partition and hence acts properly discontinuously on $S^{n}-\Lambda$. In short $\Lambda$ is the limit set of $H$. Our proof of Theorem 1.1 is done.

\section{Modified blocks}

\subsection{Partial prisms}

Let $n=2 k-1$ as in previous chapters. A convex cone in $\boldsymbol{R}^{n}$ is a closed convex subset $C \subset \boldsymbol{R}^{n}$, contained in a halfspace, which is closed under taking nonnegative linear combinations. $C$ is generated by the set $\Sigma$ if $C=\{\lambda \Sigma \mid \lambda \geq 0\}$. We call $C$ a simplex-cone if $C$ is generated by an $(n-1)$-simplex which does not contain 0 . We also insist that $C$ is $n$-dimensional.

Let $C$ be a simplex-cone. Let $H \subset \boldsymbol{R}^{n}$ be a codimension 1 hyperplane which does not contain 0 . We say that $H$ cuts $C$ if $H \cap C$ is an $(n-1)$-simplex. In this case $C$ is generated by $H \cap C$. If $H$ cuts $C$ we set $\Sigma=C \cap H$ and let $[C, H]=\{\lambda \Sigma \mid \lambda \leq 1\}$. With this definition, $[C, H]$ is an $n$-simplex, one of whose vertices is 0 , and whose other vertices are the vertices of $\Sigma$. We say that a partial prism is a set isometric to a set of the form

$$
\Pi=\operatorname{closure}\left(\left[C, H_{1}\right]-\left[C, H_{0}\right]\right) .
$$


where $H_{0}$ and $H_{1}$ cut $C$ and $\left[C, H_{0}\right] \subset\left[C, H_{1}\right]$. We call $C \cap H_{0}$ the inner boundary of $\Pi$ and we call $C \cap H_{1}$ the outer boundary of $\Pi$. Note-and this is crucial for our constructions - that the inner and outer boundaries can share vertices in common or even coincide. In all cases there is a canonical bijection between the inner boundary vertices and the outer boundary vertices: The matched vertices lie on the same line through 0 .

$\partial \Pi$ consists of two $(n-1)$-simplices - the inner and outer boundaries - and some $(n-1)$-dimensional partial prisms. This lets us define a canonical PL involution of $\Pi$ which interchanges the inner and outer boundaries. If $n=1$ then $\Pi$ is an interval or a point, and our involution reverses the interval or fixes the point depending on the case. In general the PL involution is the cone, to the center of mass of $\Pi$, of the PL involution which is defined on each partial prism of $\partial \Pi$ and which swaps inner and outer boundary components. We call this map the canonical involution.

We also can define a canonical triangulation of $\Pi$. If $\Pi$ is a simplex then we use $\Pi$ itself as the triangulation. Otherwise we triangulate $\partial \Pi$ (by induction) and then cone the resulting triangulation to the center of mass of $\Pi$. We call this the canonical triangulation of $\Pi$. The canonical PL involution is affine when restricted to each simplex in the canonical triangulation. The important point about the canonical triangulation is that it has this 2 -fold symmetry.

\subsection{Separators}

Let $n=2 k-1$ as above. Let $\langle\cdot\rangle$ be the convex hull operation. We say that a weighted simplex is an $n$-simplex $\Delta$ together with a map $S: V \Delta \rightarrow(0,1]$. Let $v_{1}, \ldots, v_{n+1}$ be the vertices of $\Delta$. Let $S_{i}=S\left(v_{i}\right)$. Let

$$
v_{i}^{*}=S_{i} v_{i}+\left(1-S_{i}\right) \beta_{S} ; \quad \beta_{S}=\frac{\sum_{i=1}^{n+1} S_{i} v_{i}}{\sum_{i=1}^{n+1} S_{i}} \in \Delta
$$

Note that $v_{i}^{*}=v_{i}$ if and only if $S_{i}=1$. In all cases $v_{i}^{*}$ is contained in the halfopen interval $\left(\beta_{S}, v_{i}\right]$ which joins $\beta_{S}$ to $v_{i}$. Hence $v_{1}^{*}, \ldots, v_{n+1}^{*}$ are in general position. Finally, we define

$$
\Delta_{S}=\left\langle\bigcup_{i=1}^{n+1} v_{i}^{*}\right\rangle ; \quad[\Delta, S]=\operatorname{closure}\left(\Delta-\Delta_{S}\right)
$$

We call $[\Delta, S]$ a separator. We call $\partial \Delta$ and $\partial \Delta_{S}$ respectively the inner and outer boundaries of $[\Delta, S]$. For each codimension 1 face $\Sigma_{S}$ of $\Delta_{S}$ there is a unique codimension 1 face $\Sigma$ of $\Delta$ such that $\Sigma_{S} \subset\left\langle\beta_{S} \cup \Sigma\right\rangle$. The set

$$
\left\langle\beta_{S} \cup \Sigma\right\rangle-\left\langle\beta_{S} \cup \Sigma_{S}\right\rangle
$$


is a partial prism. Therefore $[\Delta, S]$ is canonically partitioned into $n+1$ partial prisms.

The canonical involutions on the individual prisms piece together to produce a canonical involution of $[\Delta, S]$ which swaps $\partial \Delta$ and $\partial \Delta_{S}$. This involution is affine on each of $\partial \Delta$ and $\partial \Delta_{S}$. The canonical triangulations of the individual prisms piece together to give a canonical triangulation of the separator. The canonical involution of the separator is affine when restricted to each simplex in the canonical triangulation. The combinatorial structure of the canonical triangulation depends entirely on the set $S^{-1}(1) \subset V \Delta$. Any permutation of $V \Delta$ which respects this set extends to a PL automorphism of $[\Delta, S]$ which is affine on each simplex in the triangulation and also affine on the boundaries. This observation underlies Lemma 5.1 below.

If $\psi: \Delta \rightarrow \Delta^{\prime}$ is an affine isomorphism which carries $S$ to $S^{\prime}$ then $\psi([\Delta, S])=$ $\left[\Delta^{\prime}, S^{\prime}\right]$. Thus the separator construction is affinely natural. Also $[\Delta, S]$ varies continuously with $S$. Finally, a short computation reveals that $\beta_{S}$ is the barycenter of $\Delta_{S}$.

\subsection{Warped blocks}

Say that a weighted block is a block $\Omega$ equipped with a weighting of its vertices, $S: V \Omega \rightarrow(0,1]$. Let $(\Omega, S)$ be a weighted block. Let $\partial \Delta_{0}$ be the outer terminal of $\Omega$, so that $\Omega \subset \Delta_{0}$. Let $\partial \Delta_{1}$ and $\partial \Delta_{2}$ be the inner terminals of $\Omega$. Let $v_{1}, \ldots, v_{n+1}$ be the vertices of $\Delta_{0}$. Every point of $\Delta_{0}$ has the form

$$
x=\sum_{i=1}^{n+1} \lambda_{i} v_{i} ; \quad \text { where } \quad \sum_{i=1}^{n+1} \lambda_{i}=1 .
$$

Let $S_{i}=S\left(v_{i}\right)$. We define

$$
P_{S}(x)=\frac{\sum_{i=1}^{n+1} S_{i} \lambda_{i} v_{i}}{\sum_{i=1}^{n+1} S_{i} \lambda_{i}} \in \Delta_{0} .
$$

The map $P_{S}$ is not a linear map. However it is a projective automorphism of $\Delta_{0}$. In particular $P_{S}$ permutes the set of simplices contained in $\Delta_{0}$. We define

$$
\Omega_{S}=P_{S}(\Omega) .
$$

The terminals of $\Omega_{S}$ are the three simplex boundaries

$$
\partial \Delta_{0} ; \quad \Delta_{1, S}=P_{S}\left(\partial \Delta_{1}\right) ; \quad \Delta_{2, S}=P_{S}\left(\partial \Delta_{2}\right) .
$$


$P_{S}$ maps the triangulation of $\Omega$ to a combinatorially equivalent triangulation of $\Omega_{S}$. Thus $\Omega_{S}$ has a canonical triangulation. There is a canonical PL homeomorphism $W_{S}: \Omega \rightarrow \Omega_{S}$ which is affine on each simplex of the triangulations. $W_{S}$ conjugates the 3 -fold PL symmetry $\sigma$ of $\Omega$ to a 3 -fold PL symmetry $\sigma_{S}$ of $\Omega_{S}$. By construction $\sigma_{S}$ is affine when restricted to each of the terminals of $\Omega_{S}$.

We call $\Omega_{S}$ a warped block. The map $W_{S}$ sets up a canonical bijection between $V \Omega$ and $V \Omega_{S}$. In this way we transfer the map $S: V \Omega \rightarrow(0,1]$ to a map $S: V \Omega_{S} \rightarrow(0,1]$. In other words the vertices of $\Omega_{S}$ are naturally weighted. Note that $S$ restricts to give a weighting to each of the terminals of $\Omega_{S}$. For instance, we have the restriction map $S: V \Delta_{1, S} \rightarrow(0,1]$.

If $\left(\Omega_{1}, S_{1}\right)$ and $\left(\Omega_{2}, S_{2}\right)$ are weighted blocks and $T: \Omega_{1} \rightarrow \Omega_{2}$ is an affine map such that $S_{1}=S_{2} \circ T$ then $T\left(\Omega_{S_{1}}\right)=\Omega_{S_{2}}$. This follows from the fact that $T$ conjugates $P_{S_{1}}$ to $P_{S_{2}}$, as can be seen from Equation 21] Our warping construction is affinely natural even though the map $P_{S}$ is not itself affine.

\subsection{Main constructions}

General modified blocks Let $(\Omega, S)$ be as above. Let $\Delta_{S}$ be as in Equation 18. Let $T_{S}$ be the affine map which carries $\Delta_{S}$ to $\Delta$ in such a way that $T_{S}\left(v_{i}^{*}\right)=v_{i}$ for all $i$. Note that $T_{S}([\Delta, S])$ is a separator whose inner boundary is $\Delta$. We define

$$
[\Omega, S]=\Omega_{S} \cup T_{S}\left(\left[\Delta_{0}, S\right]\right) \cup\left[\Delta_{1, S}, S\right] \cup\left[\Delta_{2, S}, S\right] .
$$

We have attached one separator to each terminal of $\Omega_{S}$. We call $[\Omega, S]$ a general modified block. We call $\Omega_{S}$ the core of $[\Omega, S]$. Note that $[\Omega, S]$ again has three terminals; these are the free boundaries of the attached separators. The outer terminal is $T_{S}\left(\partial \Delta_{0}\right)$.

\section{Remarks}

(i) Note that $\partial \Delta_{0}$ is the inner boundary of $T_{S}\left(\left[\Delta_{0}, S\right]\right)$ whereas $\partial \Delta_{j}$ is the outer boundary of $\left[\Delta_{j}, S\right]$. From a PL standpoint this asymmetry in our construction disappears: Each separator has its canonical involution which turns it inside out.

(ii) $[\Omega, S]$ is not necessarily a subset of $S^{n}$. The problem is that the outer boundary of $T_{S}\left(\left[\Delta_{0}, S\right]\right)$ might be so large that it is not contained in one of the two unit simplices comprising $S^{n}$. This difficulty will be handled in section 5 
in an automatic way. Our construction will only use modified blocks which are contained in $S^{n}$.

(iii) Given our definitions in Equations [18] and 24, each vertex $v \in V \Omega_{S}$ corresponds to two vertices $v_{1}, v_{2} \in V[\Omega, S]$ and we have $v_{1}=v_{2}$ if and only if $S(v)=1$. The same remarks apply to $] \Omega_{+}, S[$ below.

Special modified blocks Suppose now that $\Omega_{+}$is the modular block constructed in section 2 . Then $\Omega_{+}=\operatorname{closure}\left(S^{n}-\Delta_{-}-\Delta_{1}-\Delta_{2}\right)$. This follows from the fact that the outer terminal of $\Omega_{+}$is $\partial \Delta_{+}$, and $S^{n}=\Delta_{+} \cup \Delta_{-}$. The weighting $S$ gives a map $S: V \Delta_{-} \rightarrow(0,1]$ as well as the maps $S: V \Delta_{j, S} \rightarrow(0,1]$ for $j=1,2$. We define

$$
] \Omega_{+}, S\left[=\left(\Omega_{+}\right)_{S} \cup\left[\Delta_{-}, S\right] \cup\left[\Delta_{1, S}, S\right] \cup\left[\Delta_{2, S}, S\right] .\right.
$$

The first separator is contained in $\Delta_{-}$. We call $] \Omega_{+}, S[$ a special modified block. We call $\left(\Omega_{+}\right)_{S}$ the core of $] \Omega_{+}, S[$. The free boundaries of the separators are the terminals.

\subsection{Degeneration}

Let $n_{1}<n_{2}$ be two integers. Let $\left\{\Delta_{m}\right\}$ denote a sequence of $n_{2}$-simplices. Let $\Delta^{\prime}$ be an $n_{1}$-simplex. We say that $\Delta_{m}$ converges barycentrically to $\Delta^{\prime}$ if some collection of $n_{1}$ vertices of $\Delta_{m}$ converges to the vertices of $\Delta^{\prime}$ as $m \rightarrow \infty$ and the remaining vertices of $\Delta_{m}$ converge to the barycenter of $\Delta^{\prime}$. (We shall always have a consistent labelling of the vertices.) Referring to section 4.2:

Lemma 4.1 Let $\Delta$ be an $n_{2}$-simplex and let $\Delta^{\prime}$ be an $n_{1}$-simplex face of $\Delta$. Let $S_{m}: V \Delta \rightarrow(0,1]$ and $S^{\prime}: V \Delta^{\prime} \rightarrow(0,1]$ be such that $S_{m}(v) \rightarrow S^{\prime}(v)$ if $v \in V \Delta^{\prime}$ and $S_{m}(v) \rightarrow 0$ otherwise. $\Delta_{S_{m}}$ converges barycentrically to $\Delta_{S^{\prime}}^{\prime}$.

Proof Equation 17 extends continuously to the case when some (but not all) of the $S_{i}$ are zero. Extending $S^{\prime}$ by the $0-$ map we have $S^{\prime}=\lim S_{m}$. When $S_{i}^{\prime}=0$ we have $v_{i}^{*}=\beta_{S^{\prime}}$, the barycenter of $\Delta_{S^{\prime}}^{\prime}$.

Let $\Omega_{+}^{j}$ denote the $n_{j}$-dimensional block from the Block Lemma. In general we use the notation $X^{j}$ to refer to an object associated to $\Omega_{+}^{j}$ though sometimes we simplify the notation. Referring to Equation 2 there is a natural embedding $i: S^{n_{1}} \rightarrow S^{n_{2}}$ defined by $i\left(A_{j}^{1}\right)=A_{j}^{2}$ and $i\left(C_{j}^{2}\right)=C_{j}^{2}$ for $j=1, \ldots, k_{1}$. Suppose $S^{1}: V \Omega_{+}^{1} \rightarrow(0,1]$. We define $\left(\Omega^{\prime}, S^{\prime}\right)=i\left(\Omega_{+}^{1}, S_{1}\right)$ and $\Omega=\Omega_{+}^{2}$. Suppose 
$S_{m}: V \Omega \rightarrow(0,1]$ is a sequence of maps such that $S_{m}(v) \rightarrow S^{\prime}(v)$ if $v \in V \Omega^{\prime}$ and $S_{m}(v) \rightarrow 0$ otherwise. Let $] \Omega, S_{m}\left[\right.$ and $\left[\Omega, S_{m}\right]$ be the special and general modified blocks based on $\left(\Omega, S_{m}\right)$. Say that a filled-in terminal of a modified block is a simplex bounded by a terminal. The following result is the key to Theorem 1.4

Lemma 4.2 The filled-in terminals of $] \Omega, S_{m}$ [ converge barycentrically to the filled-in terminals of $] \Omega^{\prime}, S^{\prime}[$.

Proof By Equations 18 and 25 the filled-in terminals of $] \Omega, S_{m}\left[\right.$ are $\left(\Delta_{-}^{2}\right)_{S_{m}}$ and $\left(\Delta_{j, S_{m}}^{2}\right)_{S_{m}}$. Let $\Delta^{\prime}=i\left(\Delta_{+}^{1}\right)$ and $\Delta_{-}^{\prime}=i\left(\Delta_{-}^{1}\right)$ and $\Delta_{j}^{\prime}=i\left(\Delta_{j}^{1}\right)$. The filled-in terminals of $] \Omega^{\prime}, S^{\prime}\left[\right.$ are $\left(\Delta_{-}^{\prime}\right)_{S^{\prime}}$ and $\left(\Delta_{j, S^{\prime}}^{\prime}\right)_{S^{\prime}}$. In all cases, $j \in\{1,2\}$. Now, $S_{m}, \Delta_{+}^{2}, S^{\prime}$ and $\Delta^{\prime}$ are as in Lemma 4.1. Hence $\left(\Delta_{-}^{2}\right)_{S_{m}}$ converges barycentrically to $\left(\Delta_{-}^{\prime}\right)_{S^{\prime}}$. A direct calculation (which we did numerically on examples to be sure) shows that the first $2 k_{1}$ vertices of $\Delta_{j, S_{m}}^{2}=P_{S_{m}}\left(\Delta_{j}^{2}\right)$ converge to the vertices of $\Delta_{j, S^{\prime}}^{\prime}=P_{S^{\prime}}\left(\Delta_{j}^{\prime}\right)$. Lemma 4.1 finishes the proof in this case.

\section{The rest of the results}

\subsection{Modified correspondences}

Suppose that $\Gamma$ is a modular pattern and $\Gamma^{\prime} \subset \Gamma$ is a modular sub-pattern. We define $\Gamma_{e}^{\prime}$ just as we defined $\Gamma_{e}$. We have $\Gamma_{e}^{\prime} \subset \Gamma_{e}$ for all $e \in E T$. To each $e \in E T$ we assign a pair $\Psi^{\prime}(e)=\left(\Delta_{e}, \phi_{e}\right)$, where $\Delta_{e}$ is an $n$-dimensional simplex of $S^{n}$ and $\phi_{e}: \Gamma_{e} \rightarrow V \Delta_{e}$ is a bijection. This is as in section 3.3. We say that $\Psi^{\prime}$ is a modified simplicial correspondence for the pair $\left(\Gamma^{\prime}, \Gamma\right)$ if it satisfies the Properties 1 and 3 for simplicial correspendences and

Property $2^{\prime}$ Given $e_{1}$ and $e_{2}$ in $E T$ we let $\left(\Delta_{j}, \phi_{j}\right)=\Psi^{\prime}\left(e_{j}\right)$. Suppose $v_{j}$ is a vertex of $\Delta_{j}$ for $j=1,2$. Then $v_{1}=v_{2}$ iff $\phi_{1}^{-1}\left(v_{1}\right)=\phi_{2}^{-1}\left(v_{2}\right)$ and the common object $\phi_{j}^{-1}\left(v_{j}\right)$ belongs to $\Gamma_{e}^{\prime}$.

We define the map $\Psi_{\infty}^{\prime}: S^{1} \rightarrow S^{n}$ just as in Equation [1] Lemmas 3.4, 3.6 and 3.7 work exactly the same way for $\Psi_{\infty}^{\prime}$ as they do for $\Psi_{\infty}$. Property $2^{\prime}$ causes a change in Lemma 3.5. The same argument in Lemma 3.5 proves that $\Psi^{\prime}$ identifies points on $S^{1}$ if and only if they are the common endpoints of a geodesics in $\Gamma^{\prime}$. Thus $\Psi_{\infty}^{\prime}$ factors through an embedding of $Q_{\Gamma^{\prime}}$ into $S^{n}$. 
Remark The remark at the end of section 3.4 needs to be modified in the setting here. Property $2^{\prime}$ gives a bijection between $\Gamma^{\prime} \cup V T$ and a certain subset $V^{\prime} \Psi^{\prime} \subset V \Psi^{\prime}$ of the block vertices. $\Lambda=\Psi^{\prime}\left(S^{1}\right)$ is the closure of $V^{\prime} \Psi^{\prime}$.

\section{$5.2 \quad$ Modified block networks}

Each modified block has a canonical triangulation, obtained from the triangulations on the core and on the separators. Suppose that $\Xi_{1}$ and $\Xi_{2}$ are modified blocks with symmetries $\sigma_{1}$ and $\sigma_{2}$. Let $\operatorname{Map}\left(\Xi_{1}, \Xi_{2}\right)$ be the set of triangulationrespecting PL maps from $\Xi_{1}$ to $\Xi_{2}$. Suppose that $\Xi_{j}$ has a weighted core $\left(\Omega_{j}\right)_{S_{j}}$ for $j=1,2$. We say that the bijection $\psi: V\left(\left(\Omega_{1}\right)_{S_{1}}\right) \rightarrow V\left(\left(\Omega_{2}\right)_{S_{2}}\right)$ between the core vertex sets is a perfect matching if $\psi^{-1} \circ \sigma_{2} \circ \psi=\sigma_{1}$ and $S_{2} \circ \psi=S_{1}$. In other words, $\psi$ is symmetry-respecting and weight-respecting.

Lemma 5.1 A perfect matching $\psi$ extends to an element of $\operatorname{Map}\left(\Xi_{1}, \Xi_{2}\right)$. When $\Xi_{1}$ and $\Xi_{2}$ are general modified blocks, this extension is affine if it matches up the outer terminals.

Proof The combinatorial structure of the separators of $\Xi$ only depends on $S$. The combinatorially identical triangulations on $\Xi_{1}$ and $\Xi_{2}$ define the extension of $\psi$. When the outer terminals are matched up, the extension of $\psi$ to the cores is an affine map $\widehat{\psi}$. This follows from the affine naturality of the warping process. It follows from Equation 17 that the map $\widehat{\psi}$ maps the separators of $\Xi_{1}$ to the separators of $\Xi_{2}$. This follows from the affine naturality of the separator construction.

The rest of our constructions depend on some $f \in \square(\Gamma)$, which we fix throughout the discussion. We say that the elements of $V T$ have weight 1 . This convention, together with $f$, assigns weights to each element of $\Gamma_{\tau}$, the set in Equation [14. Let $[\Omega, S]$ be a modified block. Let $\tau \in F T$ be an ideal triangle. We say that a $\tau$-labelling of $[\Omega, S]$ is a bijection $\phi: \Gamma_{\tau} \rightarrow V \Omega_{S}$ such that $\phi \circ g=\sigma \circ \phi$ and $S \circ \phi=f$. All maps above have $\Gamma_{\tau}$ as their range. As in section 3.5, the element $g$ is the order 3 stabilizer of $\tau$ which cycles the edges counterclockwise. $\sigma$ is the order 3 PL symmetry of $[\Omega, S]$. So, $\phi$ carries the weights of $f$ to the weighting of $\Omega_{S}$. We make the same definitions for $] \Omega_{+}, S[$.

We have labelled $V \Omega_{S}$, because $V[\Omega, S]$, the actual vertex set of $[\Omega, S]$, generally has more vertices than $\Gamma_{\tau}$ has elements. Here we describe an induced labelling of $V[\Omega, S]$. Let $\partial \Delta$ be one of the terminals of $[\Omega, S]$. Then one of the terminals $\partial \Delta^{\prime}$ of $\Omega_{S}$ is such that $\partial \Delta$ and $\partial \Delta^{\prime}$ form the boundary of a separator 
of $[\Omega, S]$. As with all separators, there is a canonical bijection $\beta: V \Delta \rightarrow V \Delta^{\prime}$. One of the three edges $e$ bounding $\tau$ is such that $\phi^{-1}(V \Delta)=\Gamma_{e}$. We label the vertex $\beta(v) \in V \Delta$ by the pair $\left(\phi^{-1}(v), e\right)$. In this way, each vertex of $V[\Omega, S]$ is labelled by a pair $(\gamma, e)$, where $\gamma \in \Gamma_{e}$ and $e$ is an edge of $\tau$. Given Remark (iii) in section 4.4, and our construction here, the induced labelling has the property that $v_{1}$ is labelled by a pair $\left(\gamma, e_{1}\right)$ and $v_{2}$ is labelled by a pair $\left(\gamma, e_{2}\right)$. Here $\gamma$ is the element of $\Gamma_{\tau}$ which labels $v$. We have $v_{1}=v_{2}$ if and only if $f(\gamma)=1$. We call this the separation principle.

We define modified block networks just as we defined block networks in section 3.5 , using modified blocks in place of blocks. The one twist is that $\Omega\left[t_{+}\right]$is a special modified block and all the other $\Omega[\tau]$ are general modified blocks.

Lemma 5.2 There exists a modified block network for $f \in \square(\Gamma)$.

Proof The proof is essentially the same as the one given in Lemma 3.9. Let $t_{+}=t_{0}, t_{1}, t_{2}, \ldots$ be as in Lemma 3.9. We need to construct modified blocks $\Omega_{0}, \Omega_{1}, \Omega_{2} \ldots$, where $\Omega_{j}=\Omega\left[t_{j}\right]$. We set $\left.\Omega_{0}=\right] \Omega_{+}, S[$ as we must. At the induction step we choose the affine map $A$ which takes the outer terminal of $\left[\Omega_{+}, S\right]$ to $\partial \Delta$, the terminal corresponding to the edge $e$ of $t_{v}$, in such a way as to respect the labellings.

Remark As in section 3.5 the modified block network is unique up to the choice of the $t_{0}$-labelling. However, if we base our construction on some general system of weights that is not invariant under $P S L_{2}(\boldsymbol{Z})$, as we do in the proof of Theorem 1.3 below, then there are potentially as many different geometric types of network as their are $G$ equivalence classes of edges in $E T$. Here $G$ is the symmetry group of $f$ (which we will take to be a finite index subgroup of $\left.P S L_{2}(\boldsymbol{Z})\right)$.

\subsection{Proof of Theorem 1.2}

We continue with the notation from above. We set $\Gamma^{\prime}=f^{-1}(1)$. We use our modified block network to define a modified correspondence for $\left(\Gamma^{\prime}, \Gamma\right)$ : For each edge $e \in E T$ we define $\Psi(e)=\left(\Delta_{e}, \phi_{e}\right)$, where $\Delta_{e}$ is the relevant boundary simplex of $\Omega[\tau]$ and $\phi_{e}$ is the labelling of $\Delta_{e}$ induced by the $\tau$-labelling of $\Omega[\tau]$. Here $\tau$ is one of the two ideal triangles which has $e$ in its boundary. Our construction guarantees that $\Psi^{\prime}(e)$ is the same using either choice of $\tau$. The nesting properties of the modified block network are the same as for the original 
block network. Hence $\Psi^{\prime}$ has property 3. The same argument as in section 3.6 shows that $\Psi^{\prime}$ has Property 1.

Property $2^{\prime}$ follows from the Separation Principle. To see how this works, we consider our construction from a different point of view. We start with the block network for $\Gamma$. We then warp each block in the network. This changes the geometry of the network, but none of its combinatorial structure. The warped network still has property 2. Next, we split apart the terminals and insert separators - two per terminal because the terminal includes into two warped blocks. (We like to think of this as blowing air into the terminals.) Figure 5.1 shows a schematic picture.

The separators have the effect of splitting apart vertices which are labelled by geodesics $\gamma$ in $\Gamma$ which have weight less than 1 . In the warped block network $\gamma$ labels a single vertex. After the separators are added, there is an infinite list of vertices associated to $\gamma$. Each of these vertices has a label of the form $(\gamma, e)$, where $e$ is an edge of ET crossed by $\gamma$. If $\gamma$ has weight 1, then all these infinitely many vertices coalesce into one. The separators do not affect these weight-1 vertices.

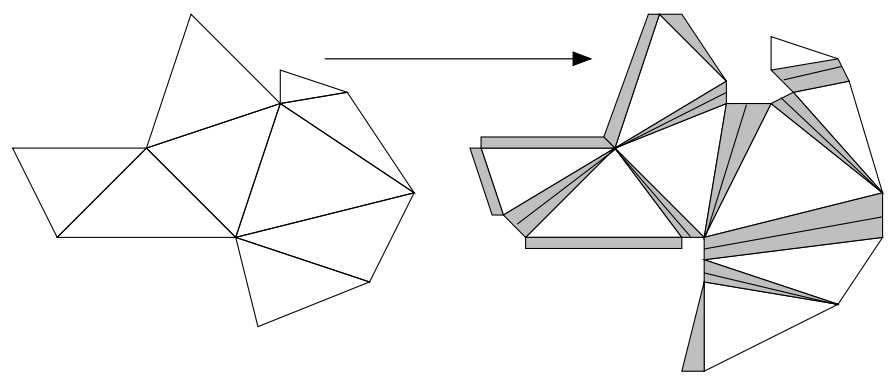

Figure 5.1

Thus our modified block network defines a modified simplicial correspondence $\Psi^{\prime}$ for $\left(\Gamma^{\prime}, \Gamma\right)$. As in section 5.1 we have our embedding $i: Q_{\Gamma^{\prime}} \rightarrow S^{n}$. We define $\Lambda_{f}=i\left(Q_{\Gamma^{\prime}}\right)$. The representation $\rho_{f}$ is constructed exactly as in section 3, with Lemma [5.1] used in place of Lemma 3.8. The same argument as in section 3 shows that $\Lambda_{f}$ is the limit set of $\rho_{f}$. The modified blocks and their symmetries are continuous functions of $f \in \square(\Gamma)$. Thus our two maps $\rho: \square(\Gamma) \rightarrow \operatorname{PL}\left(G, S^{n}\right)$ and $\Lambda: \square(\Gamma) \rightarrow\left[S^{n}\right]$ are continuous maps in the appropriate topologies.

\subsection{Proof of Theorem 1.3}

The modular group is hiding behind Theorem 1.3 
Lemma 5.3 Any cusped finite volume hyperbolic surface is homeomorphic to a quotient of the form $\boldsymbol{H}^{2} / G$, where $G$ is a finite index modular subgroup.

Proof This is a well-known result. Every cusped surface has a triangulation into ideal triangles. Each edge of an ideal triangle has a center point, the fixed point set of the isometric involution of the triangle which stabilizes that edge. We can cut apart our surface and re-glue the ideal triangles so that the center points of the edges are matched. This changes the geometric structure but not the topology. The resulting surface then develops into the hyperbolic plane, onto the modular tiling. Thus the new surface, which is homeomorphic to the original, has the form $\boldsymbol{H}^{2} / G$ with $G \subset P S L_{2}(\boldsymbol{Z})$.

By Lemma 5.3 it suffices to consider the case of Theorem 1.3 where $\Sigma=\boldsymbol{H}^{2} / G$, so that $\pi_{1}(\Sigma)=G$, a finite index modular subgroup. Let $\Gamma$ be the orbit of $\Gamma^{\prime}$ under $P S L_{2}(\boldsymbol{Z})$. Since $G$ has finite index in $P S L_{2}(\boldsymbol{Z})$, we have that $\Gamma$ is a modular pattern. We can define a modified simplicial correspondence for the pair $\left(\Gamma^{\prime}, \Gamma\right)$ even when $\Gamma^{\prime}$ does not have complete modular symmetry. The definitions and results in section 5.1 go through word for word.

Let $f: \Gamma \rightarrow\left\{\frac{1}{2}, 1\right\}$ be the map defined by the rule $f(\gamma)=1$ if $\gamma \in \Gamma^{\prime}$ and $f(\gamma)=1 / 2$ if $\gamma \in \Gamma-\Gamma^{\prime}$. Even though $f$ is not necessarily $P S L_{2}(\boldsymbol{Z})$-invariant we can define a modified block network for $f$. This network has $G$-symmetry rather than $P S L_{2}(\boldsymbol{Z})$-symmetry from the PL standpoint. The modified block network in turn defines a modified simplicial correspondence for $\left(\Gamma^{\prime}, \Gamma\right)$. The same argument as in the proof of Theorem 1.2 (which is just adapted from section 3) gives a representation $\rho: G \rightarrow \operatorname{PL}\left(S^{n}\right)$ which has $i\left(Q_{\Gamma^{\prime}}\right)$ as its limit set.

\subsection{Proof of Theorem 1.4}

Given an affine map $A$ let $\|A\|=\sup _{v}\|A(v)\|$ be the operator norm, with the sup being taken over unit vectors.

Lemma 5.4 Let $\Delta^{\prime}$ be an $n_{1}$-dimensional face of $\Delta$, the unit $n_{2}$-simplex. Let $\left\{A_{m}\right\}$ be a sequence of affine maps of $\boldsymbol{R}^{n_{2}}$, with uniformly bounded operator norm, such that $\left.A_{m}\right|_{\Delta^{\prime}}$ converges to an affine injection $A^{\prime}: \Delta^{\prime} \rightarrow \boldsymbol{R}^{n_{2}}$. Let $\left\{\Xi_{m}\right\}$ be a sequence of $n$-simplices which converge barycentrically to some $n^{\prime}$-simplex $\Xi^{\prime} \subset \Delta^{\prime}$. Then $A_{m}\left(\Xi_{m}\right)$ converges barycentrically to $A^{\prime}\left(\Xi^{\prime}\right)$. 
Proof The first $n_{1}$ vertices of $\Xi_{m}$ converge to the vertices of $\Xi^{\prime}$. The bound on the operator norms guarantees that the first $n_{1}$ vertices of $A_{m}\left(\Xi_{m}\right)$ converge to $A^{\prime}\left(\Xi^{\prime}\right)$. The remaining vertices of $\Xi_{m}$ converge to the barycenter of $\Xi^{\prime}$. Again, the bound on the operator norms guarantees the images of these remaining vertices under $A_{m}$ converge to the barycenter of $A^{\prime}\left(\Xi^{\prime}\right)$.

We continue the notation from section 4.6 and also use the notation from Theorem [1.4. Let $] \Omega, S_{m}$ [ and $\left[\Omega, S_{m}\right]$ be the special and general modified blocks based on $\Omega={ }_{2} \Omega_{+}$, corresponding to $f_{m}$. Thus $] \Omega, S_{m}$ [ is the zeroth modified block in the modified block network for $f_{m}$ and $\left[\Omega, S_{m}\right]$ is the general modified block used in the induction step of Lemma 5.2. We let $S$ be the weighting on ${ }_{1} \Omega_{+}$that correponds to $f \in \square\left(\Gamma_{1}\right)$.

For each $m$ we need to choose a $t_{+}$-labelling of $] \Omega, S_{m}[$. We pick the labelling so that the vertices in $A^{\prime} \cup C^{\prime}$ are labelled by objects associated to $\Gamma_{1}$. We can make the labellings independent of $m$, since only the weights vary with $m$. We can choose a $t_{+}$-labelling of $]_{1} \Omega, S\left[\right.$ which is consistent with our $t_{+}$-labellings of $] \Omega, S_{m}\left[\right.$. All the same remarks apply to $\left[\Omega, S_{m}\right]$ and $\left[{ }_{1} \Omega, S\right]$. This sets things up so that $] \Omega, S_{m}\left[\right.$ and $\left[\Omega, S_{m}\right]$ are as in Lemma 4.2

For each $m$ we have a modified block network $N_{m} \subset S^{n_{2}}$. We also have a modified block network $N \subset S^{n_{1}}$. Let $N^{\prime}=i(N)$. The terminals of $N_{m}$ are canonically bijective with the terminals of $N^{\prime}$. Both are indexed by ET.

Lemma 5.5 Each filled-in terminal of $N_{m}$ converges barycentrically to the corresponding terminal of $N^{\prime}$ as $m \rightarrow \infty$.

Proof Let $t_{0}, t_{1}, t_{2} \ldots$ be as in Lemma [5.2. For ease of notation we suppress the dependence on $m$. Let $\Omega_{j}$ be the modified block associated to $t_{j}$ when the construction is based on $f_{m} \in \square\left(\Gamma_{2}\right)$. Let ${ }_{1} \Omega_{j}$ be the modified block associated to $t_{j}$ when the construction is based on $f \in \square(\Gamma)$. Let $\Omega_{j}^{\prime}=i\left({ }_{1} \Omega_{j}\right)$. It suffices to show that the filled-in terminals of $\Omega_{j}$ converge barycentrically to the filled-in terminals of $\Omega_{j}^{\prime}$. For $j=0$ this is exactly Lemma 4.2 .

Suppose the result is true for $j=1, \ldots, w-1$. We consider the case $j=w$. We adopt the notation from Lemma 3.9 and 5.2 . Thus $D$ is the outer filledin terminal of $\left[\Omega_{+}, S_{m}\right]$ and $A: D \rightarrow \Delta$ is such that the two filled-in inner terminals of $\Omega_{w}$ are $A\left(\Delta_{1}\right)$ and $A\left(\Delta_{2}\right)$. Here $\Delta_{1}$ and $\Delta_{2}$ are the two inner filled-in terminals of $\left[\Omega_{+}, S_{m}\right]$. By induction $\Delta$ converges to barycentrically to one of the inner terminals of $\Omega_{v}^{\prime}$. Thus the outer filled-in terminal of $\Omega_{w}$ converges barycentrically to the outer filled-in terminal of $\Omega_{w}^{\prime}$. We just have 
to show that $A\left(\Delta_{1}\right)$ and $A\left(\Delta_{2}\right)$ converge barycentrically to the inner filled-in terminals of $\Omega_{w}^{\prime}$.

Note that $\Delta_{+} \subset D$ and either $A(D) \subset \Delta_{+}$or $A(D) \subset \Delta_{-}$. In either case $A$ maps the standard unit simplex inside an isometric copy of itself. This bounds $\|A\|$, independent of $m$. With a view towards using Lemma 5.4 we let $\Delta^{\prime}=i\left({ }_{1} \Delta_{+}\right)$. We let $\Xi_{m}$ be $\Delta_{1}$, the first inner terminal of $\Omega_{w}$. We let $\Xi^{\prime}$ be the first inner terminal $\Delta_{1}^{\prime}$ of $\Omega_{w}^{\prime}$. The inner filled-in terminals of $\left[\Omega_{+}, S\right]$ are the same as two of the terminals of $] \Omega_{+} m, S[$. Therefore, by Lemma 4.2 we have $\Xi_{m} \rightarrow \Xi^{\prime}$ barycentrically. By Lemma 5.4 we see that $A\left(\Delta_{1}\right) \rightarrow A^{\prime}\left(\Delta_{1}^{\prime}\right)$ barycentrically. But $A^{\prime}\left(\Delta_{1}^{\prime}\right)$ is one of the inner filled-in terminals of $\Omega_{w}$. The same argument works for $\Delta_{2}$. This completes the induction step.

It follows from Lemma 5.5 that the limit sets $\Lambda_{f_{m}}$ converge to $i(\Lambda)$ and in fact the maps $S^{1} \rightarrow \Lambda_{f_{m}}$ converge pointwise to the map $S^{1} \rightarrow \Lambda_{f}$. The action of $\rho_{f_{m}}$ on $\Lambda_{f_{m}}$ is determined by the embedding $S^{1} \rightarrow \Lambda_{f_{m}}$ and by the action of $P S L_{2}(\boldsymbol{Z})$ on $\partial \boldsymbol{H}^{2}$. Hence the restriction of $\rho_{f_{m}}$ to $\Lambda_{f_{m}}$ converges to the restriction of $i \circ \rho_{f} \circ i^{-1}$ to $\Lambda_{f}$. This completes the proof of Theorem 1.4, except in the case when $\Gamma_{1}$ is the empty pattern.

The empty pattern To deal with the empty pattern we first define the standard representation of $P S L_{2}(\boldsymbol{Z})$ onto $S^{1}$. We think of the unit interval $I_{+}$ as the convex hull of the vectors $A=(1,0)$ and $C=(0,1)$ in $\boldsymbol{R}^{2}$. The midpoint of $I_{+}$is the vector $B=(1 / 2,1 / 2)$. Let $I_{1+}=\langle A \cup B\rangle$ and $I_{2+}=\langle B \cup C\rangle$. Here $\langle\cdot\rangle$ denotes the convex hull operation. Let $I_{-}$be another copy of $I+$. Let $S^{1}=I_{+} \cup I_{-}$. Let $\sigma_{+} \in \operatorname{PL}\left(S^{1}\right)$ be the order 3 element whose action is given by the orbit $I_{-} \rightarrow I_{1+} \rightarrow I_{2+}$ and $A \rightarrow B \rightarrow C$. Let $\iota \in \operatorname{PL}\left(S^{1}\right)$ be the order 2 element whose action is given by the orbit $I_{+} \rightarrow I_{-}$and $A \rightarrow C$. The elements $\sigma_{+}$and $\iota$ generate an action on $S^{1}$ which is topologically conjugate to the standard action of $P S L_{2}(\boldsymbol{Z})$ on $\partial \boldsymbol{H}^{2}$. To see this, we identify $A, B$, and $C$ with the vertices of the ideal triangle $t_{+}$. Then $\sigma_{+}$acts on $S^{1}$ just as the order 3 stabilizer of $t_{+}$acts on $\partial \boldsymbol{H}^{2}$ and $\iota$ acts on $S^{1}$ just as the order 2 stabilizer of one of the edges of $t_{+}$acts on $\partial \boldsymbol{H}^{2}$.

When $\Gamma_{1}$ is the empty pattern we are dealing with a sequence $\left\{f_{m}\right\}$ which converges to the 0 -map. In this case, the same analysis as that given in Lemma 4.2 shows that the terminals of $] \Omega, S_{m}$ [ converge to the line segments $I_{-}, I_{1+}$ and $I_{2+}$. The same argument we give in Lemma 5.5 then shows that $\Lambda_{f_{m}}$ converges to $S^{1}$ and the restriction of $\rho_{f_{m}}$ to $\Lambda_{f_{m}}$ converges to the standard representation. This completes our proof of Theorem 1.4. 


\section{References}

[1] L Bers, On Boundaries of Teichmuller Spaces and on Kleinian Groups I, Annals of Math 91 570-600 (1970)

[2] M Bonk, O Schramm, Embeddings of Gromov Hyperbolic Spaces, Geom. Funct. Anal. 10 (2000) 266-306

[3] F Gardiner, Teichmuller Theory and Quadratic Differentials, Wiley Interscience (1987)

[4] B Maskit, Kleinian Groups, Springer-Verlag (1987)

[5] J G Ratcliff, Foundations of Hyperbolic Manifolds, Graduate Texts in Mathematics 149, Springer-Verlag (1994)

[6] C Series, Geometric Methods of Symbolic Coding, from: "Ergodic Theory, Symbolic Dynamics, and Hyperbolic Spaces" (Bedford, Keane and Series, editors) Oxford Science Publications (1991)

[7] R E Schwartz, Degenerating the Complex Hyperbolic Ideal Triangle Groups, Acta Mathematica 186 (2001)

[8] R E Schwartz, Circle Quotients and String Art, Topology 41 (2001) 495-523

[9] RE Schwartz, Pappus's Theorem and the Modular Group, IHES Publications Mathematiques 78 (1993) 